\title{
A Vlasov description of the Euler equation
}

\author{
Peter Smereka \\ Department of Mathematics, University of Michigan, Ann Arbor, MI 48109, USA
}

Received 6 June 1995, in final form 4 March 1996

Recommended by R Krasny

\begin{abstract}
A form of the Euler equation using an impulse formulation is presented. This form is based on a representation of the divergence-free projection operator in terms of a continuous distribution of vortex dipoles which have a finite self-induced velocity. A generalization of the Euler equation is presented as a kinetic equation similar to the Vlasov-Poisson equation. An interesting feature of this generalization of the Euler equation is that it has nontrivial solutions in one space dimension. The stability of the spatially homogeneous solution is also studied. Distribution functions with a single maximum are found to be linearly stable, whereas those with two maxima can be unstable and the initial value problem ill-posed. Weak solutions of this kinetic equation are found using a water-bag model and a simple model of inviscid 1D turbulence is developed.
\end{abstract}

AMS classification scheme numbers: 76C99, 76E99

\section{Introduction}

Recently, there has been interest in the Euler equation written in terms of a momentum variable, $\boldsymbol{p}$, which we shall call the impulse density. In this variable the Euler equation can be written as

$$
\frac{\partial \boldsymbol{p}}{\partial t}+\boldsymbol{u} \cdot \nabla \boldsymbol{p}=-(\nabla \boldsymbol{u})^{T} \boldsymbol{p}
$$

where the liquid velocity, $\boldsymbol{u}$, is the divergence-free projection of the impulse. The Euler equation written in this form has been studied by a number of investigators. Sagdeev et al [1] and Tur and Yanovsky [4] derive this equation based mainly on kinematic considerations. They also use this equation to find new topological invariants for the Euler equation. Oseledets[3] and Kuz'min [2] both show that this equation is a Hamiltonian system with respect to a certain Lie-Poisson bracket.

The above equation was used, in two space dimensions, by Krasny [8] in a vortexdipole sheet model of a wake. He solved this equation using a Lagrangian numerical scheme analogous to the vortex-blob method. Buttke [5] (see also Buttke and Chorin [6]) devised a similar Lagrangian numerical scheme. This scheme was implemented in three space dimensions and is discretely Hamiltonian. Buttke also provides numerical evidence that the method converges.

Maddocks and Pego [7] provide a new unconstrained Hamiltonian form of the Euler equation. In this derivation they recover the aforementioned form of the impulse equation as well as an alternative form which appears to have advantages in situations with a free boundary. Chorin and Buttke [6] call equation (1) the Euler equation written in 
magnetization variables. Chorin [9] has used this 'magnet' representation of the Euler equation to study turbulent states and their connection with phase transitions.

The outline of this paper is as follows. In section 2, a derivation of a more general impulse equation which reflects a gauge freedom of (1) is presented. It is then demonstrated that the divergence-free projection operator can be split into two parts: a local and a nonlocal part. The nonlocal part is determined from a continuous distribution of vortex dipoles. The local part is seen to be the self-induced velocity of a vortex dipole. It is crucial that the vortex dipoles have a self-induced velocity if the velocity field produced is to be divergence-free.

Next, it is shown by an appropriate choice of gauge, that force density can be written in terms of derivatives of the nonlocal field only. Based on this observation a kinetic description of the Euler equation is proposed, where the impulse is replaced with a distribution function. If the distribution function converges to a delta function, our kinetic equation reduces to the Euler equation. Therefore we see that our kinetic equation is a generalization of the Euler equation and contains the Euler equation as a solution for special initial data. This kinetic equation may also contain some physical meaning for other initial data; for example, it could be interpreted as a coarse-grained description of a highly turbulent flow of an inviscid liquid. The distribution function then gives the statistics of the impulse within the averaging volume.

An interesting feature of this kinetic equation is that it has nontrivial solutions in one space dimension when the distribution function is not simply a Dirac mass. If our equation is indeed a course-grained description of inviscid turbulence, some 1D turbulence models may be studied. We observe that a spatially homogeneous distribution function is a solution of our kinetic equation and examine its stability in section 4 . It is found that if the distribution function has a single maximum, the solution is linearly stable. On the other hand, if the distribution function has two maxima, conditions necessary for the onset of instability are found. It is also shown that if the problem is unstable, the initial value problem is ill-posed. It is conjectured that this ill-posedness results in the formation of singularities which may be connected to intermittency. In section 5 we construct solutions to the 1D Vlasov equation using the water-bag model of DePackh [18] and develop a very simple model of 1D inviscid turbulence. An interesting feature of this model is the formation of finite time singularities. It is important to note that these singularities are unrelated to finite time singularities of the Euler equation.

\section{Impulse formulation}

We begin by writing the Euler equation as

$$
\frac{\partial \boldsymbol{\omega}}{\partial t}-\nabla \times(\boldsymbol{u} \times \boldsymbol{\omega})=0
$$

where $\boldsymbol{u}$ is the velocity of the liquid and $\boldsymbol{\omega}=\nabla \times \boldsymbol{u}$ is the vorticity. Next, we introduce a vector field, $\boldsymbol{p}$, which satisfies

$$
\nabla \times \boldsymbol{p}=\boldsymbol{\omega} .
$$

If we substitute (3) into (2) we find that the time evolution of $\boldsymbol{p}$ is given by

$$
\frac{\partial \boldsymbol{p}}{\partial t}-\boldsymbol{u} \times(\nabla \times \boldsymbol{p})=\nabla \psi
$$

for some function, $\psi$, which can be thought of as a gauge. We shall call $p$ the impulse. If we pick $\psi=-\boldsymbol{u} \cdot \boldsymbol{p}+\lambda$, we can write (4) as

$$
\frac{\partial \boldsymbol{p}}{\partial t}+\boldsymbol{u} \cdot \nabla \boldsymbol{p}=-(\nabla \boldsymbol{u})^{T} \boldsymbol{p}+\nabla \lambda
$$


which in component form is

$$
\frac{\partial p_{\alpha}}{\partial t}+u_{\beta} \frac{\partial p_{\alpha}}{\partial x_{\beta}}=-p_{\beta} \frac{\partial u_{\beta}}{\partial x_{\alpha}}+\frac{\partial \lambda}{\partial x_{\alpha}}
$$

where $\alpha$ and $\beta$ denote the Cartesian components with summation convention over repeated Greek subscripts. Equation (5), with $\lambda=0$, is the impulse form of the Euler equation given in the introduction. In a later section we will derive another version of the impulse equation.

\subsection{Relation between velocity and impulse}

Since $\nabla \times \boldsymbol{p}=\nabla \times \boldsymbol{u}$, it follows that

$$
\boldsymbol{p}=\boldsymbol{u}+\nabla \phi
$$

where $\phi$ satisfies the relation

$$
\Delta \phi=\nabla \cdot p
$$

and $\Delta$ denotes the Laplacian. Equation (7) follows from (6) and also because the velocity field of the liquid is divergence-free $(\nabla \cdot \boldsymbol{u}=0)$. We solve (6) and (7) for $\boldsymbol{u}$ in terms of $\boldsymbol{p}$ to obtain

$$
\boldsymbol{u}=\mathcal{B} \boldsymbol{p}
$$

where $\mathcal{B}$ is the projection operator

$$
\mathcal{B}=I-\nabla \Delta^{-1} \nabla .
$$

$\mathcal{B} \boldsymbol{p}$ is the projection of $\boldsymbol{p}$ onto divergence-free vector fields.

\subsection{Physical meaning of impulse}

Consider a fluid of infinite extent with a vorticity distribution supported on $\Omega$. It is known that the total momentum of the liquid is not well defined since the velocity field decays like $r^{-3}$. This gives rise to a conditionally convergent integral whose value depends on how it is evaluated. The physically important quantity is instead the impulse. It is the impulse that has the property of acting like the momentum of the fluid (see [10], p 518). This is defined in three space dimensions as

$$
\boldsymbol{I}=\frac{1}{2} \int_{\Omega} \boldsymbol{x} \times \boldsymbol{\omega} \mathrm{d} \boldsymbol{x}
$$

Since $\boldsymbol{\omega}=\nabla \times \boldsymbol{p}$ it then follows, after integration by parts, that

$$
\boldsymbol{I}=\int_{\Omega} \boldsymbol{p} \mathrm{d} \boldsymbol{x} .
$$

Therefore, $\boldsymbol{p}$ is the impulse density. We have chosen $\boldsymbol{p}$ to have the same support as $\boldsymbol{\omega}$ (which can always be done). 


\subsection{Divergence-free projection}

We first make the following definitions:

$$
\begin{aligned}
& \kappa=\frac{d-1}{d} \\
& \mathbf{B}(\boldsymbol{r})=-\frac{1}{c_{d}|\boldsymbol{r}|^{d}}\left(I-d \frac{\boldsymbol{r} \boldsymbol{r}^{T}}{|\boldsymbol{r}|^{2}}\right)
\end{aligned}
$$

where $d=2$ or 3 is the space dimension, $c_{2}=2 \pi$, and $c_{3}=4 \pi$. We also define the principal value integral to be

$$
f=\lim _{\varepsilon \rightarrow 0} \int_{|x-y|>\varepsilon} .
$$

Theorem. The divergence-free projection operator, $\mathcal{B}$, in $\mathbb{R}^{d}, d=2$ or 3 can be written as

$$
\mathcal{B} \boldsymbol{p}(\boldsymbol{x}, t)=\kappa \boldsymbol{p}(\boldsymbol{x}, t)+\boldsymbol{w}(\boldsymbol{x}, t)
$$

where

$$
\boldsymbol{w}(\boldsymbol{x}, t)=f \mathbf{B}(\boldsymbol{x}-\boldsymbol{y}) \boldsymbol{p}(\boldsymbol{y}, t) \mathrm{d} \boldsymbol{y} .
$$

This shows that the divergence-free projection can be split into a local part, $\kappa \boldsymbol{p}$, and a nonlocal part, $\boldsymbol{w}$. This theorem will be proved below.

The divergence-free projection written in the form given by (12) has an interesting interpretation. To see this we first consider

$$
u=\mathrm{B}(r) p .
$$

This is the velocity field given by a source-sink doublet located at the point $\boldsymbol{r}=0$ for an inviscid irrotational fluid with a dipole moment of $\boldsymbol{p}$ (see [10], p 89). Let $\boldsymbol{u}_{v}$ be the fluid velocity produced by a vortex ring of radius $a$ located at $r=0$. We let the impulse of the vortex ring be $\boldsymbol{p}$ ( $\boldsymbol{p}$ will be perpendicular to the face of the ring). One then finds (see [10], p 518 or [12])

$$
\boldsymbol{u}_{v}(\boldsymbol{r}) \rightarrow \mathbf{B}(\boldsymbol{r}) \boldsymbol{p} \quad \text { as } \frac{|\boldsymbol{r}|}{a} \rightarrow \infty .
$$

Therefore (14) is the velocity field produced by an infinitesimally small vortex ring with impulse $\boldsymbol{p}$, called a vortex dipole by Chefranov [13]. We shall henceforth adopt this name. It is now evident that a divergence-free velocity field can be written as a continuous distribution of vortex dipoles which have a self-induced velocity of $\kappa \boldsymbol{p}$.

Proof of theorem. We begin the proof of the theorem by writing the solution of (7) as

$$
\phi(\boldsymbol{x})=\int G_{d}(\boldsymbol{x}-\boldsymbol{y}) \frac{\partial p_{\beta}}{\partial y_{\beta}} \mathrm{d} \boldsymbol{y}
$$

where $G_{d}$ is the Green's function for the Laplacian in $\mathbb{R}^{d}, d=2$ or 3 . These are

$$
G_{2}(\boldsymbol{r})=\frac{1}{2 \pi} \log |\boldsymbol{r}|
$$

and

$$
G_{3}(r)=-\frac{1}{4 \pi|r|} .
$$


Since $\partial_{\alpha} G_{d}$ has an integrable singularity we can integrate by parts and write

$$
\phi(\boldsymbol{x})=-\int p_{\beta}(\boldsymbol{y}) \frac{\partial}{\partial y_{\beta}} G_{d}(\boldsymbol{x}-\boldsymbol{y}) \mathrm{d} \boldsymbol{y} .
$$

Hence

$$
\frac{\partial \phi}{\partial x_{\alpha}}=-\frac{\partial}{\partial x_{\alpha}} \int p_{\beta}(\boldsymbol{y}) \frac{\partial}{\partial y_{\beta}} G_{d}(\boldsymbol{x}-\boldsymbol{y}) \mathrm{d} \boldsymbol{y} .
$$

It is necessary to bring the $\partial_{\alpha}$ inside the integral. This cannot be done for (18) in the form given as $\partial_{\alpha \beta}^{2} G_{d}$ has a non-integrable singularity. To remedy this we first make the following definition:

$$
b_{a}(r)= \begin{cases}1 & r<a \\ 0 & r \geqslant a\end{cases}
$$

and note that

$$
\int b_{a}(|\boldsymbol{x}-\boldsymbol{y}|) \frac{\partial}{\partial y_{\beta}} G_{d}(\boldsymbol{x}-\boldsymbol{y}) \mathrm{d} \boldsymbol{y}=0 .
$$

Equations (18) and (20) are combined to give

$$
\frac{\partial \phi}{\partial x_{\alpha}}=-\frac{\partial}{\partial x_{\alpha}} \int\left[p_{\beta}(\boldsymbol{y})-b_{a}(|\boldsymbol{x}-\boldsymbol{y}|) p_{\beta}(\boldsymbol{x})\right] \frac{\partial}{\partial y_{\beta}} G_{d}(\boldsymbol{x}-\boldsymbol{y}) \mathrm{d} \boldsymbol{y} .
$$

We can now bring the $\partial_{\alpha}$ inside the integral and write the above equation as

$$
\partial_{\alpha} \phi(x)=T_{\alpha}^{(1)}+T_{\alpha}^{(2)}
$$

where

$$
T_{\alpha}^{(1)}=\int\left[p_{\beta}(\boldsymbol{y})-b_{a}(|\boldsymbol{x}-\boldsymbol{y}|) p_{\beta}(\boldsymbol{x})\right] \frac{\partial^{2}}{\partial y_{\alpha} \partial y_{\beta}} G_{d}(\boldsymbol{x}-\boldsymbol{y}) \mathrm{d} \boldsymbol{y}
$$

and

$$
\begin{gathered}
T_{\alpha}^{(2)}=-\int \frac{\partial}{\partial x_{\alpha}}\left[p_{\beta}(\boldsymbol{y})-b_{a}(|\boldsymbol{x}-\boldsymbol{y}|) p_{\beta}(\boldsymbol{x})\right] \frac{\partial}{\partial y_{\beta}} G_{d}(\boldsymbol{x}-\boldsymbol{y}) \mathrm{d} \boldsymbol{y} \\
=\frac{\partial p_{\beta}(\boldsymbol{x})}{\partial x_{\alpha}} \int b_{a}(|\boldsymbol{x}-\boldsymbol{y}|) \frac{\partial}{\partial y_{\beta}} G_{d}(\boldsymbol{x}-\boldsymbol{y}) \mathrm{d} \boldsymbol{y} \\
\quad+p_{\beta}(\boldsymbol{x}) \int \frac{\partial}{\partial x_{\alpha}} b_{a}(|\boldsymbol{x}-\boldsymbol{y}|) \frac{\partial}{\partial y_{\beta}} G_{d}(\boldsymbol{x}-\boldsymbol{y}) \mathrm{d} \boldsymbol{y} .
\end{gathered}
$$

The first term in $T_{\alpha}^{(2)}$ is zero by (20). For the second term we consider $d=3$, let $\boldsymbol{r}=\boldsymbol{y}-\boldsymbol{x}$, and use (17) to obtain

$$
T_{\alpha}^{(2)}=-\frac{p_{\beta}(\boldsymbol{x})}{4 \pi} \int \frac{r_{\alpha} r_{\beta}}{r^{4}} \frac{\mathrm{d}}{\mathrm{d} r} b_{a}(r) \mathrm{d} \boldsymbol{r}
$$

where $r=|r|$. It follows from the definition of $b_{a}$ that

$$
\frac{\mathrm{d}}{\mathrm{d} r} b_{a}(r)=-\delta(r-a)
$$

where $\delta$ is the Dirac delta function; therefore (22) becomes

$$
T_{\alpha}^{(2)}=\frac{p_{\beta}(\boldsymbol{x})}{4 \pi} \int_{|r|=a} \frac{r_{\alpha} r_{\beta}}{r^{4}} \mathrm{~d} S=\frac{1}{3} p_{\alpha}(\boldsymbol{x}) .
$$

A similar calculation shows

$$
T_{\alpha}^{(2)}=\frac{1}{2} p_{\alpha}(\boldsymbol{x})
$$


for $d=2$. Thus, for both cases we can write

$$
T_{\alpha}^{(2)}=\frac{1}{d} p_{\alpha}(\boldsymbol{x}) .
$$

Let us now turn our attention to $T_{\alpha}^{(1)}$, which can be written as

$$
\begin{array}{r}
T_{\alpha}^{(1)}=\int_{|\boldsymbol{x}-\boldsymbol{y}| \leqslant a}\left[p_{\beta}(\boldsymbol{y})-p_{\beta}(\boldsymbol{x})\right] \frac{\partial^{2}}{\partial y_{\alpha} \partial y_{\beta}} G_{d}(\boldsymbol{x}-\boldsymbol{y}) \mathrm{d} \boldsymbol{y} \\
+\int_{|\boldsymbol{x}-\boldsymbol{y}|>a} p_{\beta}(\boldsymbol{y}) \frac{\partial^{2}}{\partial y_{\alpha} \partial y_{\beta}} G_{d}(\boldsymbol{x}-\boldsymbol{y}) \mathrm{d} \boldsymbol{y} .
\end{array}
$$

Since

$$
\int_{\varepsilon<|\boldsymbol{x}-\boldsymbol{y}|<a} \frac{\partial^{2}}{\partial y_{\alpha} \partial y_{\beta}} G_{d}(\boldsymbol{x}-\boldsymbol{y}) \mathrm{d} \boldsymbol{y}=0
$$

it follows that

$$
T_{\alpha}^{(1)}=Q+\int_{|\boldsymbol{x}-\boldsymbol{y}|>\varepsilon} p_{\beta}(\boldsymbol{y}) \frac{\partial^{2}}{\partial y_{\alpha} \partial y_{\beta}} G_{d}(\boldsymbol{x}-\boldsymbol{y}) \mathrm{d} \boldsymbol{y}
$$

where

$$
Q=\int_{|x-y| \leqslant \varepsilon}\left[p_{\beta}(\boldsymbol{y})-p_{\beta}(\boldsymbol{x})\right] \frac{\partial^{2}}{\partial y_{\alpha} \partial y_{\beta}} G_{d}(\boldsymbol{x}-\boldsymbol{y}) \mathrm{d} \boldsymbol{y} .
$$

We expand $p_{\beta}(\boldsymbol{y})$ in a Taylor series to obtain

$$
Q=\int_{|\boldsymbol{r}|<\varepsilon}\left[\frac{\partial p_{\beta}(\boldsymbol{x})}{\partial x_{\mu}} r_{\mu}+\frac{1}{2} \frac{\partial^{2} p_{\beta}(\boldsymbol{x})}{\partial x_{\mu} \partial x_{v}} r_{\mu} r_{\nu}+\mathcal{O}\left(|\boldsymbol{r}|^{3}\right)\right] \frac{\partial^{2}}{\partial y_{\alpha} \partial y_{\beta}} G(\boldsymbol{r}) \mathrm{d} \boldsymbol{r} .
$$

The linear terms of the Taylor expansion vanish upon integration and we find

$$
Q=c_{1} \varepsilon^{2}+\mathcal{O}\left(\varepsilon^{3}\right)
$$

where $c_{1}$ depends on the second derivatives of $\boldsymbol{p}$ at $\boldsymbol{x}$. Next, we let $\varepsilon \rightarrow 0$ to obtain

$$
\frac{\partial \phi(\boldsymbol{x})}{\partial x_{\alpha}}=\frac{1}{d} p_{\alpha}(\boldsymbol{x})+f p_{\beta}(\boldsymbol{y}) \frac{\partial^{2}}{\partial y_{\alpha} \partial y_{\beta}} G_{d}(\boldsymbol{x}-\boldsymbol{y}) \mathrm{d} \boldsymbol{y} .
$$

Substitution of (27) into (6) gives (12).

Remark. Oseledets [3] and Kuz'min [2] have also introduced representations of the velocity field in terms of a continuous distribution of vortex dipoles. They both have expressions similar to (12), namely

$$
\mathcal{B} \boldsymbol{p}=\mu \boldsymbol{p}+\int \mathbf{B}(\boldsymbol{x}-\boldsymbol{y}) \boldsymbol{p}(\boldsymbol{y}) \mathrm{d} \boldsymbol{y}
$$

where Oseledets takes $\mu=0$ and Kuz'min takes $\mu=1$. The difficulty with both of these expressions is that $\mathbf{B}(\boldsymbol{r})$ has a non-integrable singularity at $\boldsymbol{r}=0$, which means (28) is not well defined. This difficulty is alleviated by (12) because the integral is taken as a principal value. Furthermore, Oseledets derives the Lagrangian form of the impulse equation by implicitly taking the principal value integral while keeping $\mu=0$ to obtain Chefranov's [13] interacting vortex dipoles (which have no self-induced velocity). This work shows that Chefranov's work cannot be considered a Lagrangian description of the impulse equation. Even with the self-induced velocity included it is unlikely that Chefranov's equations will converge to the impulse equation [14]. Buttke's approach does not suffer from this difficulty because he regularizes $\mathbf{B}$. 


\subsection{Alternative impulse equation}

In view of (12) we can write

$$
\boldsymbol{u}(\boldsymbol{x}, t)=\kappa \boldsymbol{p}(\boldsymbol{x}, t)+\boldsymbol{w}(\boldsymbol{x}, t) .
$$

If we substitute (29) into (5) and take† $\lambda=(\kappa / 2)|p|^{2}$, we obtain

$$
\frac{\partial \boldsymbol{p}}{\partial t}+\boldsymbol{u} \cdot \nabla \boldsymbol{p}=-(\nabla \boldsymbol{w})^{T} \boldsymbol{p} .
$$

We notice that (30) can be written as $\dot{\boldsymbol{p}}=\boldsymbol{F}$ where the denotes the material derivative and $\boldsymbol{F}=-(\nabla \boldsymbol{w})^{T} \boldsymbol{p}$. In this form the force acting on a vortex dipole is $-(\nabla \boldsymbol{w})^{T} \boldsymbol{p}$. Therefore the force on the dipole is determined by its impulse and the gradient of the nonlocal part of the velocity field. This indicates that there is no self-force on the vortex dipole in this gauge.

\section{Kinetic theory}

An interesting feature of the Euler equation given by (30) is the form of $\boldsymbol{w}(\boldsymbol{x})$; the velocity induced at $\boldsymbol{x}$ by the vortex dipoles not located at $\boldsymbol{x}$. Equation (30) implies that the force on a dipole arises only from the nonlocal field; this indicates that in the gauge we have chosen there is no self-force on the dipoles. The velocity field created by the vortex dipoles decays like $r^{-3}$ which is a long range interaction. Therefore we see that the motion of the vortex dipoles is analogous to the motion of electrons which is modelled by the Vlasov-Poisson equation. With this in mind we will derive a Vlasov-type equation for (30); first, however, we will write down the Vlasov-Poisson equation to make the analogy more transparent.

Let $f(\boldsymbol{x}, \boldsymbol{v}, t)$ be the probability density of the electron having position $\boldsymbol{x}$ and velocity $\boldsymbol{v}$. The time evolution of $f(\boldsymbol{x}, \boldsymbol{v}, t)$ is given by

$$
\frac{\partial f}{\partial t}+\boldsymbol{v} \cdot \frac{\partial f}{\partial \boldsymbol{x}}+\boldsymbol{F} \cdot \frac{\partial f}{\partial \boldsymbol{v}}=0 .
$$

$\boldsymbol{F}=\boldsymbol{F}(\boldsymbol{x}, t)$ is the force field given by

$$
\boldsymbol{F}=-q \nabla \phi
$$

where $q$ is the charge of an electron and $\phi$ is the electric potential. The electric potential is

$$
\phi(x)=\int G_{3}(\boldsymbol{x}-\boldsymbol{y}) \rho(\boldsymbol{y}, t) \mathrm{d} \boldsymbol{y}
$$

where $\rho(\boldsymbol{x}, t)$ is the expected excess charge density

$$
\rho(\boldsymbol{x}, t)=q\left(\int f(\boldsymbol{x}, \boldsymbol{v}, t) \mathrm{d} \boldsymbol{v}-1\right) .
$$

For our Vlasov form of the Euler equation we let $f(\boldsymbol{x}, \boldsymbol{p}, t)$ be the probability density of a fluid particle having position $\boldsymbol{x}$ and impulse $\boldsymbol{p}$. The distribution function, $f$, is then evolved according to the following equation:

$$
\frac{\partial f}{\partial t}+\frac{\partial}{\partial \boldsymbol{x}} \cdot(\boldsymbol{u} f)+\frac{\partial}{\partial \boldsymbol{p}} \cdot(\boldsymbol{F} f)=0
$$

where $\boldsymbol{u}$ and $\boldsymbol{F}$ are the velocity and force field, respectively. In this situation, (33) is analogous to (13) which is now written as

$$
\boldsymbol{w}(\boldsymbol{x}, t)=f \mathbf{B}(\boldsymbol{x}-\boldsymbol{y}) \boldsymbol{j}(\boldsymbol{y}, t) \mathrm{d} \boldsymbol{y}
$$

$\dagger$ This choice of gauge was suggested by R E Caflisch. 
where

$$
\boldsymbol{j}(\boldsymbol{x}, t)=\int \boldsymbol{p} f(\boldsymbol{x}, \boldsymbol{p}) \mathrm{d} \boldsymbol{p} .
$$

Equation (37) is comparable to (34). We can think of $\boldsymbol{w}$ as a vector potential. In view of (12) we can write

$$
\boldsymbol{w}=\mathcal{B} \boldsymbol{j}-\kappa \boldsymbol{j} .
$$

With $\boldsymbol{w}$ now defined the velocity and the force fields are given, respectively, by

$$
\boldsymbol{u}=\kappa \boldsymbol{p}+\boldsymbol{w}
$$

and

$$
\boldsymbol{F}=-(\nabla \boldsymbol{w})^{T} \boldsymbol{p}
$$

(refer to (29) and (30)). Since

$$
\frac{\partial}{\partial \boldsymbol{x}} \cdot \boldsymbol{u}+\frac{\partial}{\partial \boldsymbol{p}} \cdot \boldsymbol{F}=0
$$

(35) simplifies to

$$
\frac{\partial f}{\partial t}+\boldsymbol{u} \cdot \frac{\partial f}{\partial \boldsymbol{x}}+\boldsymbol{F} \cdot \frac{\partial f}{\partial \boldsymbol{p}}=0 .
$$

Therefore our Vlasov description for the vortex dipoles consists of (41) along with (37)-(40).

Next, the connection of (41) with (30) will be examined. The ensemble average of the liquid velocity, $\overline{\boldsymbol{u}}$, is defined using

$$
n(\boldsymbol{x}, t) \overline{\boldsymbol{u}}(\boldsymbol{x}, t)=\int \boldsymbol{u} f(\boldsymbol{x}, \boldsymbol{p}, t) \mathrm{d} \boldsymbol{p}
$$

where

$$
n(\boldsymbol{x}, t)=\int f(\boldsymbol{x}, \boldsymbol{p}, t) \mathrm{d} \boldsymbol{p} .
$$

We shall show that $\overline{\boldsymbol{u}}$ is divergence-free provided the initial data for the distribution function satisfies

$$
\int f(\boldsymbol{x}, \boldsymbol{p}, 0) \mathrm{d} \boldsymbol{p}=1 .
$$

To prove this statement we take the zeroth moment of (41), and find

$$
\frac{\partial n}{\partial t}+\nabla \cdot(n \overline{\boldsymbol{u}})=0
$$

where $\overline{\boldsymbol{u}}$ is found to be

$$
n \overline{\boldsymbol{u}}=\mathcal{B}(n \overline{\boldsymbol{p}}) .
$$

It follows from (44) and (43) that $\partial n / \partial t=0$. We recall from (42) that $n(\boldsymbol{x}, 0)=1$; therefore, $\nabla \cdot \overline{\boldsymbol{u}}=0$ and $n(\boldsymbol{x}, t)=1$. This means we have

$$
\begin{aligned}
& \boldsymbol{j}=\overline{\boldsymbol{p}} \\
& \overline{\boldsymbol{u}}=\mathcal{B} \overline{\boldsymbol{p}} .
\end{aligned}
$$

Hence, the average velocity, $\overline{\boldsymbol{u}}$, is divergence-free.

In view of the above we can consider (41) a kinetic description of the Euler equation provided the initial conditions for the distribution function satisfy (42). Another way to view (41) is as a generalization of the Euler equation, where the impulse, $\boldsymbol{p}(\boldsymbol{x}, t)$, has been 
replaced by a probability distribution, $f(\boldsymbol{x}, \boldsymbol{p}, t)$. If $f(\boldsymbol{x}, \boldsymbol{p}, t)$ converges to $\delta(\boldsymbol{p}-\overline{\boldsymbol{p}}(\boldsymbol{x}, t))$, the impulse at $\boldsymbol{x}$ will be equal to $\overline{\boldsymbol{p}}$ with probability one. In this case $\overline{\boldsymbol{p}}(\boldsymbol{x}, t)$ should satisfy the Euler equation. We shall now verify this and look for a weak solution of the form

$$
f(\boldsymbol{x}, \boldsymbol{p}, t)=\delta(\boldsymbol{p}-\overline{\boldsymbol{p}}(\boldsymbol{x}, t)) .
$$

It is clear that the initial condition of (47) satisfies (42). Substituting (47) into (41), we find

$$
\nabla \delta(\boldsymbol{p}-\overline{\boldsymbol{p}}) \cdot\left[\frac{\partial \overline{\boldsymbol{p}}}{\partial t}+(\kappa \boldsymbol{p}+\boldsymbol{w}) \cdot \frac{\partial \overline{\boldsymbol{p}}}{\partial \boldsymbol{x}}+(\nabla \boldsymbol{w})^{T} \boldsymbol{p}\right]=0 .
$$

In order for the above equation to be satisfied $\overline{\boldsymbol{p}}$ must satisfy (30). Therefore we see that a special solution of (41) is the incompressible Euler equation. It is conceivable that with initial data other than Dirac masses, solutions of (41) will have physical meaning. One possibility is that (41) may represent a homogenized or coarse-grained description for a turbulent flow, with $f(\boldsymbol{x}, \boldsymbol{p}, t)$ acting as a probability distribution function. More precisely, let $\boldsymbol{p}(\boldsymbol{x}, t)$ be the solution of (30) and consider the averaged or smoothed quantity,

$$
\langle\boldsymbol{p}(\boldsymbol{x}, t)\rangle=\int K(\boldsymbol{x}-\boldsymbol{y}) \boldsymbol{p}(\boldsymbol{y}, t) \mathrm{d} \boldsymbol{y}
$$

where $K$ is a positive function with compact support and unit mass. The support of $K$ can be considered the coarsening volume. On the other hand, one can define the averaged quantity,

$$
\overline{\boldsymbol{p}}(\boldsymbol{x}, t)=\int f(\boldsymbol{x}, \boldsymbol{p}, t) \boldsymbol{p} \mathrm{d} \boldsymbol{p}
$$

in terms of the distribution function. If $f(\boldsymbol{x}, \boldsymbol{p}, t)$ represents the distribution of the impulse density, $\boldsymbol{p}$, within the coarsening volume centred at $\boldsymbol{x}$, it then follows that

$$
\overline{\boldsymbol{p}}(\boldsymbol{x}, t)=\langle\boldsymbol{p}(\boldsymbol{x}, t)\rangle .
$$

It is our hope that (41) is a qualitative description of $f(\boldsymbol{x}, \boldsymbol{p}, t)$. To see this possibility, let us return to (30) and let $\boldsymbol{p}=\langle\boldsymbol{p}\rangle+\boldsymbol{p}^{\prime}$ and write $\boldsymbol{w}$ as

$$
\boldsymbol{w}=f \mathbf{B}(\boldsymbol{x}-\boldsymbol{y})\left[\langle\boldsymbol{p}(\boldsymbol{y}, t)\rangle+\boldsymbol{p}^{\prime}(\boldsymbol{y}, t)\right] \mathrm{d} \boldsymbol{y} .
$$

Since $\int K(\boldsymbol{x}-\boldsymbol{y}) \boldsymbol{p}^{\prime}(\boldsymbol{y}, t) \mathrm{d} \boldsymbol{y}=0$, it is conceivable for a rapidly fluctuating impulse density that

$$
\boldsymbol{w} \approx f \mathbf{B}(\boldsymbol{x}-\boldsymbol{y})\langle\boldsymbol{p}(\boldsymbol{y}, t)\rangle \mathrm{d} \boldsymbol{y} .
$$

If we let $f$ be the distribution of the impulse density within the averaging volume we have that $\langle\boldsymbol{p}\rangle$ is given by (49); therefore, we find $\boldsymbol{w}$ to be approximately given by (36). Given this approximation, we can write (30) as

$$
\dot{\boldsymbol{p}}=-(\nabla \boldsymbol{w})^{T} \boldsymbol{p} \quad \text { where } \boldsymbol{w}=f \int \mathbf{B}(\boldsymbol{x}-\boldsymbol{y}) \boldsymbol{p} f(\boldsymbol{y}, \boldsymbol{p}, t) \mathrm{d} \boldsymbol{p} \mathrm{d} \boldsymbol{y} .
$$

The time evolution of $f(\boldsymbol{x}, \boldsymbol{p}, t)$ can be found by using a self-consistent field approach (e.g. Liboff [15], p 157). This gives (41).

Other important averaged quantities, defined in terms of the distribution function, are the average fluid velocity,

$$
\overline{\boldsymbol{u}}(\boldsymbol{x}, t)=\int \boldsymbol{u} f(\boldsymbol{x}, \boldsymbol{p}, t) \mathrm{d} \boldsymbol{p}
$$

and the turbulent kinetic energy,

$$
\theta(\boldsymbol{x}, t)=\frac{1}{2} \int|\overline{\boldsymbol{u}}-\boldsymbol{u}|^{2} f(\boldsymbol{x}, \boldsymbol{p}, t) \mathrm{d} \boldsymbol{p} .
$$


Remark. Brenier [11] has also introduced a kinetic description of the Euler equation, which is as follows:

$$
\frac{\partial f}{\partial t}+\boldsymbol{v} \cdot \frac{\partial f}{\partial \boldsymbol{x}}+\boldsymbol{E} \cdot \frac{\partial f}{\partial \boldsymbol{v}}=0
$$

where $\boldsymbol{E}=-\nabla P$ and $P$ is chosen to satisfy the incompressibility condition

$$
\int f \mathrm{~d} v=1
$$

To determine $P$, we relax the above condition and let

$$
\rho(\boldsymbol{x}, t)=\int f \mathrm{~d} \boldsymbol{v} .
$$

The zeroth moment of (53) gives, in component form,

$$
\partial_{t} \rho+\partial_{\alpha} \int f v_{\alpha} \mathrm{d} \boldsymbol{v}=0 .
$$

We multiply (53) by $\boldsymbol{v}$ and integrate over velocity space to obtain

$$
\partial_{t} \int f v_{\alpha} \mathrm{d} \boldsymbol{v}+\partial_{\beta} \int f v_{\alpha} v_{\beta} \mathrm{d} \boldsymbol{v}-E_{\alpha} \rho=0 .
$$

From these two relations we find

$$
\partial_{t t}^{2} \rho=\partial_{\alpha} \partial_{\beta} \int f v_{\alpha} v_{\beta}-\partial_{\alpha}\left(E_{\alpha} \rho\right) .
$$

We see that $\rho=1$ if $P$ satisfies the following:

$$
-\partial_{\alpha} \partial_{\alpha} P=\partial_{\alpha} \partial_{\beta} \int f v_{\alpha} v_{\beta} \mathrm{d} \boldsymbol{v} .
$$

We can see that (53) is also a generalization of the Euler equation. If we look for a solution of the form $f(\boldsymbol{x}, \boldsymbol{v}, t)=\delta(\boldsymbol{v}-\overline{\boldsymbol{v}}(\boldsymbol{x}, t))$, we see that $\overline{\boldsymbol{v}}$ will satisfy the usual Euler equation written in terms of the velocity and pressure. Equation (54) will reduce to the usual pressure equation.

\subsection{One-dimensional solutions}

An interesting class of solutions occurs when we consider initial conditions that depend only on one space variable with the average impulse also in this direction, say $x$; therefore,

$$
f(\boldsymbol{x}, \boldsymbol{p}, 0)=f(x, \boldsymbol{p}, 0) \quad \text { and } \quad \boldsymbol{j}=\left(j_{x}, 0,0\right)
$$

where the subscript $x$ denotes the $x$-component. We find that (41) has a solution of the form $f=f(x, \boldsymbol{p}, t)$ and $j=\left(j_{x}(x, t), 0,0\right)$, where $f$ satisfies

$$
\frac{\partial f}{\partial t}+\kappa\left(p_{x}-j_{x}\right) \frac{\partial f}{\partial x}-\frac{\partial w_{x}}{\partial x} p_{x} \frac{\partial f}{\partial p_{x}}=0 .
$$

In this case (38) simplifies to $\boldsymbol{w}=-\kappa \boldsymbol{j}$. It is convenient to define the averaged quantity

$$
\bar{f}\left(x, p_{x}, t\right)=\int f\left(x, p_{x}, p_{y}, p_{z}, t\right) \mathrm{d} p_{y} \mathrm{~d} p_{z}
$$

which is found to satisfy the kinetic equation

$$
\frac{\partial f}{\partial t}+u \frac{\partial f}{\partial x}+F \frac{\partial f}{\partial p}=0
$$


with

$$
u=\kappa(p-j) \quad \text { and } \quad F=\kappa p \frac{\partial j}{\partial x}
$$

where

$$
j=\int p f \mathrm{~d} p .
$$

For the sake of convenience we have dropped the bar and the $x$ subscript. The constraint on the initial conditions becomes

$$
\int f(x, p, 0) \mathrm{d} p=1 .
$$

Equation (56) must be satisfied in order to have the incompressibility condition fulfilled. It is clear from (55) that $f(x, p, t)=f_{0}(p)$ is a spatially homogeneous solution. It should also be noted that for these one-dimensional solutions $\bar{u}=0$.

The incompressibility condition (56) permits an interesting class of solutions. Let $f_{0}(p)$ satisfy (56), we can then verify that the following is a solution of (55):

$$
f(x, p, t)=f_{0}(p+\theta(x, t))
$$

provided

$$
\frac{\partial \theta}{\partial t}+\kappa\left(\theta-j_{0}\right) \frac{\partial \theta}{\partial x}=0
$$

where $j_{0}=\int f_{0}(p) p \mathrm{~d} p$ and $\theta(x, 0)$ is a bounded differentiable function. It is crucial that $f_{0}$ satisfy (56) in order for (57) to be a solution of (55). Clearly, the solutions given by (57) may exist only for finite time since the equation for $\theta(x, t)$ is Burgers' equation in a frame moving with speed $-\kappa j_{0}$. This means that $\partial_{x} \theta$ can become infinite in finite time after which (57) is no longer a classical solution of (55). It may be possible to construct weak solutions of (55) after the classical solution breaks down. We shall see below that these solutions play a role in the linear stability analysis of the spatially uniform solution.

\section{Linear analysis}

In this section we study the linear stability of a spatially homogeneous solution in the spatially periodic case for (55). We observe that any time-independent, spatially homogeneous density function, $f_{0}(p)$, is a solution of (55). We shall consider $f_{0}(p)$ that vanish as $|p| \rightarrow \infty$ and decay fast enough so that

$$
\int p^{2} f_{0}(p) \mathrm{d} p<\infty
$$

The linearized equation for $g=f-f_{0}$ is

$$
\frac{\partial g}{\partial t}+\kappa\left[\left(p-j_{0}\right) \frac{\partial g}{\partial x}+p \frac{\partial j}{\partial x} \frac{\partial f_{0}}{\partial p}\right]=0
$$

where

$$
j(x, t)=\int g(x, p, t) p \mathrm{~d} p \quad \text { and } \quad j_{0}=\int f_{0}(p) p \mathrm{~d} p .
$$

In view of (42), we have

$$
\int f_{0}(p) \mathrm{d} p=1 \quad \text { and } \quad \int g(x, p, 0) \mathrm{d} p=0 .
$$


One can verify that (58) preserves the mass; therefore,

$$
\int g(x, p, t) \mathrm{d} p=0 \quad \text { for } t>0 .
$$

It is easily demonstrated that (58) has solutions similar to (57), namely

$$
g(x, p, t)=\phi(x, t) \frac{\partial f_{0}}{\partial p} \quad \text { where } \frac{\partial \phi}{\partial t}-\kappa j_{0} \frac{\partial \phi}{\partial x}=0
$$

for any $\phi(x, 0)$. We recognize $g(x, p, t)$ as the linearization of (57). It is convenient for us to use the following independent variables:

$$
t^{*}=\kappa t \quad \text { and } \quad x^{*}=x-j_{0} t .
$$

If we substitute these variables into (58) and drop the asterisks, we have

$$
\frac{\partial g}{\partial t}+p \frac{\partial g}{\partial x}+p \frac{\partial j}{\partial x} \frac{\partial f_{0}}{\partial p}=0 .
$$

We write

$$
g(x, p, t)=\sum_{k=-\infty}^{\infty} g_{k}(p, t) \mathrm{e}^{\mathrm{i} k x}
$$

because $g(x, p, t)$ is periodic in space. The time evolution of the Fourier coefficients is then

$$
\frac{\partial g_{k}}{\partial t}+\mathrm{i} k p\left(g_{k}+j_{k} \frac{\partial f_{0}}{\partial p}\right)=0
$$

where

$$
j_{k}(t)=\int g_{k}(p, t) p \mathrm{~d} p .
$$

Equation (62) has a steady solution of the form

$$
g_{k}(p)=c_{k} \frac{\partial f_{0}}{\partial p}
$$

for arbitrary $c_{k}$. This solution corresponds to (60) and is now steady because we are in a frame moving with speed $-\kappa j_{0}$.

We shall study the stability of the spatially homogeneous solution by examining the initial value problem for (62). In our analysis we shall consider $f_{0}(p)$ and $g_{k}(p, 0)$ to be functions that are analytic in a strip of width $\delta$ of the real line.

Our approach to the initial value problem will closely follow Landau's treatment for the Vlasov-Poisson equation (see, for example, [16] or [17]). We start by taking the Laplace transform of (62) to obtain

$$
G_{k}(s)=\frac{1}{\mathrm{i} k} \frac{g_{k}(p, 0)}{p-\mu}-\frac{p J_{k}(s)}{p-\mu} \frac{\partial f_{0}(p)}{\partial p}
$$

where $\mu=\mathrm{is} / k$ and

$$
G_{k}(p, s)=\int_{0}^{\infty} \mathrm{e}^{-s t} g_{k}(p, t) \mathrm{d} t \quad \text { and } \quad J_{k}(s)=\int G_{k} p \mathrm{~d} p .
$$

We multiply by $p$, integrate both sides of the above equation, and solve for $J_{k}$ to obtain

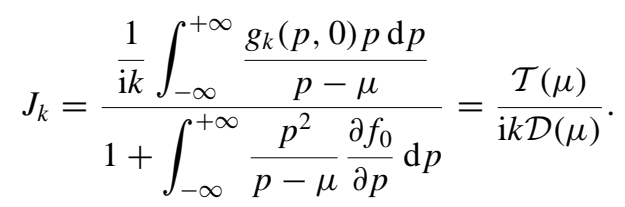


Thus, we can write

$$
G_{k}(s)=\frac{1}{\mathrm{i} k(p-\mu)}\left[g_{k}(p, 0)-\frac{p \mathcal{T}(\mu)}{\mathcal{D}(\mu)} \frac{\partial f_{0}}{\partial p}\right] .
$$

It then follows that $g_{k}(t)$ is determined by taking the inverse Laplace transform; hence,

$$
g_{k}(t)=\frac{1}{2 \pi \mathrm{i}} \int_{\gamma-\mathrm{i} \infty}^{\gamma+\mathrm{i} \infty} G_{k}(s) \mathrm{e}^{s t} \mathrm{~d} s
$$

where $\gamma$ is chosen to be to the right of all the poles of $G_{k}(s)$ in the complex $s$-plane.

In the following we shall take $k>0$; the case $k<0$ is treated similarly and yields the same stability criteria. Formally $G_{k}(s)$ is defined only for $\operatorname{Re}(s) \geqslant \gamma$. It is useful to analytically continue $G_{k}(s)$ to the left of $\operatorname{Re}(s)=\gamma$. Both $\mathcal{T}(\mu)$ and $\mathcal{D}(\mu)$ are analytic functions of $\mu$, provided that $\operatorname{Im}(\mu) \neq 0(\operatorname{Re}(s) \neq 0)$. Therefore $G_{k}(s)$ is meromorphic for $\operatorname{Re}(s)>0$. We must now analytically continue $G_{k}(s)$ into the left-half of the complex $s$-plane which is the same as analytically continuing into the lower-half of the complex $\mu$-plane. This will be done using the method developed by Landau in which $\mathcal{T}(\mu)$ and $\mathcal{D}(\mu)$ are treated separately. When $\operatorname{Im}(\mu)>0$, the integrals that appear in $\mathcal{T}(\mu)$ and $\mathcal{D}(\mu)$ are performed along the real axis. When $-\delta<\operatorname{Im}(\mu) \leqslant 0$, we deform the contour as shown in figure 1. Thus, we redefine $\mathcal{D}$ and $\mathcal{T}$ as

$$
\mathcal{D}(\mu)=1+\int_{\mathcal{C}_{L}} \frac{p^{2}}{p-\mu} \frac{\partial f_{0}}{\partial p}(p) \mathrm{d} p
$$

and

$$
\mathcal{T}(\mu)=\int_{\mathcal{C}_{L}} \frac{p g_{k}(p, 0)}{p-\mu} \mathrm{d} p
$$

where $\mathcal{C}_{L}$ denotes the Landau contour. As a result of this procedure $\mathcal{D}(\mu)$ and $\mathcal{T}(\mu)$ are now analytic functions for $\operatorname{Im}(\mu)>-\delta$. This means we have analytically continued $G_{k}(s)$ for $\operatorname{Re}(s)>-\delta k$. In the region $\operatorname{Im}(\mu)>-\delta(\operatorname{Re}(s)>-\delta k)$ the only singularities for $G_{k}(s)$ are poles which occur at $s=-\mathrm{i} k p$ and at the roots of $\mathcal{D}(\mu)=0$.

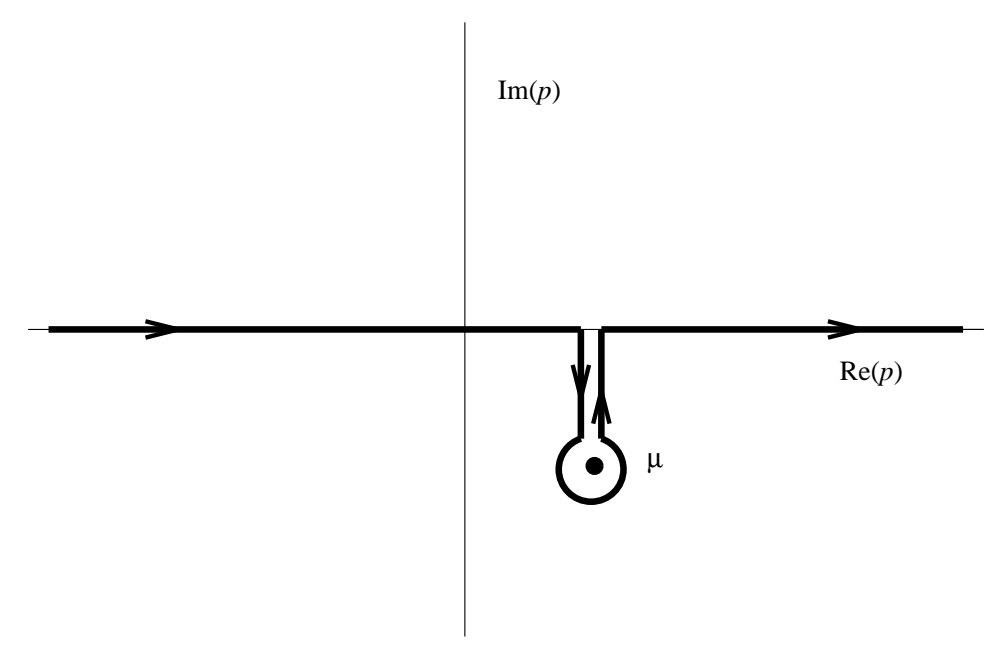

Figure 1. The Landau contour for $\operatorname{Im}(\mu)<0$. 
Next, we deform the contour given in (65) into the left-half plane for $\gamma=-k \delta$ and pick up contributions from the poles of $G_{k}$. We now have

$$
g_{k}(t)=\sum_{m=0}^{N} a_{m}+\frac{1}{2 \pi \mathrm{i}} \int_{-k \delta-\mathrm{i} \infty}^{-k \delta+\mathrm{i} \infty} G_{k}(s) \mathrm{e}^{s t} \mathrm{~d} s
$$

where $a_{m}$ are the residues of the poles at $s=s_{m}$ of $G_{k}$. We will show below that $\mathcal{T}(\mu)$ has a simple zero at $\mu=0$ and $\mathcal{D}(\mu)$ has a double zero at $\mu=0$. Thus $G_{k}$ will have a simple pole at $s=0$. Furthermore, we shall also consider $f_{0}$ so that the other zeros of $\mathcal{D}$ are simple. Let $\mu_{m}, m=1$ to $M$ denote the roots of $\mathcal{D}(\mu)=0$ such that $\operatorname{Im}(\mu)>-k \delta$. The relevant poles of $G_{k}$ are then located in the complex $s$-plane at

$$
s=-\mathrm{i} k p \quad s=0 \quad \text { and } \quad s=-\mathrm{i} k \mu_{m} \quad m=1 \text { to } M .
$$

By computing the residues of the poles, we find

$$
g_{k}(t)=A_{0}+A_{1} \mathrm{e}^{-\mathrm{i} k p t}+\sum_{j=1}^{M} B_{j} \mathrm{e}^{-\mathrm{i} k \mu_{j} t}+C
$$

where

$$
\begin{aligned}
& A_{0}=\frac{2 \mathcal{T}^{\prime}(0)}{\mathcal{D}^{\prime \prime}(0)} \frac{\partial f_{0}}{\partial p} \\
& A_{1}=g_{k}(p, 0)-\frac{p \mathcal{T}(p)}{\mathcal{D}(p)} \frac{\partial f_{0}}{\partial p} \\
& B_{j}=\frac{p}{p-\mu_{j}} \frac{\mathcal{T}\left(\mu_{j}\right)}{\mathcal{D}^{\prime}\left(\mu_{j}\right)} \frac{\partial f_{0}}{\partial p} \\
& C=\frac{1}{2 \pi \mathrm{i}} \int_{-k \delta-\mathrm{i} \infty}^{-k \delta+\mathrm{i} \infty} G_{k}(s) \mathrm{e}^{s t} \mathrm{~d} s .
\end{aligned}
$$

$A_{0}$ is time independent and corresponds to steady solutions found previously. $A_{1} \mathrm{e}^{-\mathrm{i} k p t}$ represents travelling wave solutions which propagate with speed $p$. The next term, $B_{j} \mathrm{e}^{-\mathrm{i} k \mu_{j} t}$, represents solutions that can grow or decay. This mode will decay if $\operatorname{Im}\left(\mu_{j}\right)<0$ and grow if $\operatorname{Im}\left(\mu_{j}\right)>0$. It is evident that $C$ will decay as least as fast as $\mathrm{e}^{-k \delta t}$. Therefore, it follows that if all the zeros of $\mathcal{D}(\mu)$ are in the lower-half plane, the spatially homogeneous solution, $f_{0}(p)$, is linearly stable. On the other hand, if there is at least one zero in the upper-half plane, $f_{0}(p)$ is linearly unstable. Furthermore, since the modes grow like $\mathrm{e}^{-\mathrm{i} k \mu t}$, the initial value problem for (61) is ill-posed. The situation when $f_{0}$ is linearly unstable indicates that the spatially uniform solution is unstable and a small perturbation can grow into a solution which is not spatially uniform. It is not difficult to see that in the unstable case the turbulent kinetic energy will also grow. The turbulent kinetic energy is independent of the gauge and observable. This means that the instability is physical (within the confines of the model) and not just a result of our choice of gauge.

\subsection{Stability results}

Here we will examine the stability of the spatially homogeneous solution, $f_{0}(p)$. We shall prove that if it has only a single maximum it will then be linearly stable. We also find that if $f_{0}(p)$ has more than one maximum, the initial value problem can be ill-posed and we present a simple criterion to determine stability.

Our results are obtained by recognizing $\mathcal{D}(\mu)$ to be an upper-analytic function $(\mathcal{D}$ is analytic for $\operatorname{Im} \mu>0)$. We then search for zeros in the upper-half plane of $\mathcal{D}(\mu)$ using 
the argument principle. If we find a zero of $\mathcal{D}(\mu)$ with $\operatorname{Im} \mu>0, f_{0}(p)$ is not stable and the initial value problem is ill-posed. If $\mathcal{D}(\mu)$ has no zero with $\operatorname{Im} \mu>0 f_{0}(p)$ is linearly stable.

We begin by considering a closed curve, denoted $\mathcal{C}$, in the upper-half of the complex $\mu$-plane. Since $\mathcal{D}$ is upper-analytic, the argument principle states

$$
\frac{1}{2 \pi \mathrm{i}} \int_{\mathcal{C}} \frac{1}{\mathcal{D}} \frac{\partial \mathcal{D}}{\partial \mu} \mathrm{d} \mu=M
$$

where $M$ is the number of zeros enclosed by $\mathcal{C}$. $M$ is also the winding number, with respect to the origin, of the closed curve $\mathcal{C}_{\mathcal{D}}$ which is the image of $\mathcal{C}$ under $\mathcal{D}$. Since it is true that

$$
\mathcal{D}(\mu)=1 \quad \text { as }|\mu| \rightarrow \infty
$$

it follows that the total number of zeros of $D(\mu)$ in the upper-half plane is given by the number of times the image of the real axis wraps around the origin. Let $\mathcal{C}$ henceforth denote the real axis of the complex $\mu$-plane; the winding number of the image of $\mathcal{C}$ under $\mathcal{D}$ is then

$$
M=\frac{1}{2 \pi \mathrm{i}} \int_{-\infty}^{\infty} \frac{\mathcal{D}^{\prime}\left(\mu_{R}\right)}{\mathcal{D}\left(\mu_{R}\right)} \mathrm{d} \mu_{R} .
$$

The image of the real axis under $\mathcal{D}$ is given by

$$
\mathcal{C}_{\mathcal{D}}=\mathcal{D}(\mathcal{C})=\left\{\mathcal{D}_{R}\left(\mu_{R}\right)+\mathrm{i} \mathcal{D}_{I}\left(\mu_{R}\right) \mid-\infty<\mu_{R}<\infty\right\} .
$$

In other words, $\mathcal{C}_{\mathcal{D}}$ is a closed curve in the complex $\mathcal{D}$-plane. By examining the qualitative behaviour of $\mathcal{C}_{\mathcal{D}}$ we shall compute its winding number.

We start by evaluating $\mathcal{D}(\mu)$ on the real axis of the complex $\mu$-plane. We find

$$
\begin{aligned}
\lim _{\mu_{I} \rightarrow 0^{+}} \mathcal{D}(\mu) & =1+f_{-\infty}^{\infty} \frac{p^{2}}{p-\mu_{R}} \frac{\partial f_{0}}{\partial p} \mathrm{~d} p+\mathrm{i} \pi \mu_{R}^{2} \frac{\partial f_{0}}{\partial p}\left(\mu_{R}\right) \\
& =\mathcal{D}_{R}\left(\mu_{R}\right)+\mathrm{i} \mathcal{D}_{I}\left(\mu_{R}\right)
\end{aligned}
$$

where $f$ denotes the principal value integral (see, for example, [17]).

Let us now examine the behaviour of $\mathcal{D}$ near $\mu=0$. It is clear that $\mathcal{D}_{I}(0)=0$ and $\mathcal{D}_{I}^{\prime}(0)=0$, while

$$
\mathcal{D}_{R}(0)=1+\int_{-\infty}^{\infty} p \frac{\partial f_{0}}{\partial p} \mathrm{~d} p
$$

and is found to be zero using integration by parts and (59). It also follows that $\mathcal{D}_{R}^{\prime}(0)=0$. Therefore $\mathcal{D}(0)=\mathcal{D}^{\prime}(0)=0$, implying that $\mu=0$ is a zero of $\mathcal{D}$ of at least multiplicity two. It also follows that

$$
\mathcal{D}^{\prime \prime}(0)=2 f_{-\infty}^{\infty} \frac{1}{p} \frac{\partial f_{0}}{\partial p} \mathrm{~d} p+2 \mathrm{i} \pi \frac{\partial f_{0}}{\partial p}(0) .
$$

If $\partial_{p} f_{0}(0) \neq 0$ then $\operatorname{Im}\left[D^{\prime \prime}(0)\right] \neq 0$ indicating that the multiplicity of $\mu=0$ is exactly two. On the other hand, if $\partial_{p} f_{0}(0)=0$ then $\operatorname{Im}\left[\mathcal{D}^{\prime \prime}(0)\right]=0$ and $\operatorname{Re}\left[\mathcal{D}^{\prime \prime}(0)\right]$ may or may not be zero. We now compute $\mathcal{D}^{\prime \prime}(0)$ when $\operatorname{Im}\left[\mathcal{D}^{\prime \prime}(0)\right]=0$ since it will be of use later; equation (75) can then be written as

$$
\mathcal{D}^{\prime \prime}(0)=2 \int_{-\infty}^{\infty} \frac{1}{p} \frac{\partial}{\partial p}\left[f_{0}(p)-f_{0}(0)\right] \mathrm{d} p .
$$

If we integrate by parts we find that

$$
\mathcal{D}^{\prime \prime}(0)=-2 \int_{-\infty}^{\infty} \frac{1}{p^{2}}\left[f_{0}(0)-f_{0}(p)\right] \mathrm{d} p .
$$


The two aforementioned integrals are defined without using the principal value since $\partial_{p} f_{0}(0)=0$. A similar approach shows that $\mathcal{T}(0)=0$.

Since $\mathcal{D}$ has a double zero for $\mu=0$, it follows that $\mathcal{C}_{\mathcal{D}}$ has a cusp at $\left(\mathcal{D}_{R}, \mathcal{D}_{I}\right)=(0,0)$. The angle the cusp makes with the line $\mathcal{D}_{I}=0, \mathcal{D}_{R}>0$ is denoted $\theta_{c}$ and is given by

$$
\tan \theta_{c}=\mathcal{D}_{I}^{\prime \prime}(0) / \mathcal{D}_{R}^{\prime \prime}(0) \text {. }
$$

We next examine the behaviour of $\mathcal{D}\left(\mu_{R}\right)$ as $\left|\mu_{R}\right| \rightarrow \infty$. Our first observation is that

$$
\mathcal{D}_{R}\left(\mu_{R}\right)=1+\frac{2 j_{0}}{\mu_{R}}+\frac{3 m_{2}}{\mu_{R}^{2}}+\mathcal{O}\left(\mu_{R}^{-3}\right) \quad \text { as }\left|\mu_{R}\right| \rightarrow \infty
$$

where $m_{2}=\int p^{2} f_{0}(p) \mathrm{d} p>0$. Equation (78) follows from arguments similar to those found in Nicholson [17]. It is clear from (74) that $\lim _{\left|\mu_{R}\right| \rightarrow \infty} \mathcal{D}_{I}\left(\mu_{R}\right)=0$; furthermore, as $\mu_{R} \rightarrow+\infty, \mathcal{D}_{I}\left(\mu_{R}\right)<0$ and as $\mu_{R} \rightarrow-\infty, \mathcal{D}_{I}\left(\mu_{R}\right)>0$. Thus $\mathcal{C}_{\mathcal{D}}$ must cross the line $\mathcal{D}_{I}=0$ at $\mathcal{D}_{R}=1$. We shall next consider two special cases.

Case 1. $f_{0}(p)$ has a single maximum at $p_{0}$. Here we show that this solution is linearly stable. When $f_{0}(p)$ has a single maximum, $\mathcal{D}^{\prime \prime}(0) \neq 0$ and $\mathcal{D}(0)=0$ with multiplicity two. This follows from (75), for if $p_{0} \neq 0$ then $\operatorname{Im}\left(\mathcal{D}^{\prime \prime}(0)\right) \neq 0$. If $p_{0}=0$ it follows from (76) that $\mathcal{D}^{\prime \prime}(0)<0$. We have shown above (see (78)) that the curve $\mathcal{C}_{\mathcal{D}}$ crosses the line $\mathcal{D}_{I}=0$ at $D_{R}=1$. Since $\partial_{p} f_{0}(p)$ only vanishes for finite $p$ at $p=p_{0}\left(\operatorname{Im}\left[\mathcal{D}\left(p_{0}\right)\right]=0\right), \mathcal{C}_{\mathcal{D}}$ can only cross the line $\mathcal{D}_{I}=0$ once more at the following point:

$$
\begin{aligned}
\mathcal{D}_{R}\left(p_{0}\right) & =1+\int_{-\infty}^{\infty} \frac{p^{2}}{p-p_{0}} \frac{\partial f_{0}}{\partial p} \mathrm{~d} p \\
& =p_{0}^{2} \int_{-\infty}^{\infty} \frac{1}{p-p_{0}} \frac{\partial f_{0}}{\partial p} \mathrm{~d} p \\
& =-p_{0}^{2} \int_{-\infty}^{\infty} \frac{\left[f_{0}\left(p_{0}\right)-f_{0}(p)\right]}{\left(p-p_{0}\right)^{2}} \mathrm{~d} p \leqslant 0
\end{aligned}
$$

where the equality occurs when $p_{0}=0$. It follows then that $\mathcal{C}_{\mathcal{D}}$ crosses the line $\mathcal{D}_{I}=0$ in two places, $\mathcal{D}_{R}=\mathcal{D}_{R}\left(p_{0}\right)$ and 1. This indicates that for $p_{0} \neq 0$ the curve $\mathcal{C}_{\mathcal{D}}$ wraps around the origin once and thus $M=1$. If $p_{0}=0$ one can then show $\theta_{c}=\pi$, indicating that again $M=1$. Hence, $\mathcal{D}(\mu)$ has one zero with $\operatorname{Im}(\mu) \geqslant 0$. This zero, however, is the double zero at $\mu=0$. The winding number for this zero is 1 instead of 2 because $\mathcal{C}_{\mathcal{D}}$ passes through the origin. Therefore $\mathcal{D}(\mu)$ has no zeros with $\operatorname{Im}(\mu)>0$ when $f_{0}(p)$ has a single maximum, indicating the spatially homogeneous solution is linearly stable.

Case 2. $f_{0}(p)$ has two maxima at $p_{1}$ and $p_{2}$ with $f_{0}\left(p_{1}\right) \geqslant f_{0}\left(p_{2}\right)$ and a single minimum at $p_{0}$. Here we show the condition for instability is

$$
\int_{-\infty}^{\infty} \frac{\left[f_{0}\left(p_{0}\right)-f_{0}(p)\right]}{\left(p-p_{0}\right)^{2}} \mathrm{~d} p<0
$$

and

$$
\int_{-\infty}^{\infty} \frac{\left[f_{0}\left(p_{2}\right)-f_{0}(p)\right]}{\left(p-p_{2}\right)^{2}} \mathrm{~d} p>0 .
$$

If these conditions are not satisfied then $f_{0}$ is linearly stable. We begin by noting that $\partial_{p} f_{0}$ will vanish at $p_{0}, p_{1}$, and $p_{2}$; therefore, $\mathcal{C}_{\mathcal{D}}$ will cross the line $\mathcal{D}_{I}=0$ at $\mathcal{D}_{R}=\mathcal{D}_{R}\left(p_{0}\right)$, $\mathcal{D}_{R}\left(p_{1}\right), \mathcal{D}_{R}\left(p_{2}\right)$, and 1 . From (79) we find

$$
D_{R}\left(p_{i}\right)=-p_{i}^{2} \int_{-\infty}^{\infty} \frac{\left[f_{0}\left(p_{i}\right)-f_{0}(p)\right]}{\left(p-p_{i}\right)^{2}} \mathrm{~d} p \quad \text { for } i=0,1 \text { and } 2 .
$$


We first take $p_{1} \neq 0, p_{1}<p_{2}$ with $\mathcal{D}_{R}\left(p_{2}\right) \neq 0$ and $D_{R}\left(p_{0}\right) \neq 0$. Let us consider $\mathcal{C}_{\mathcal{D}}$ as $\mu_{R}$ is increased from $-\infty$. $\mathcal{C}_{\mathcal{D}}$ starts on the real axis at $\mathcal{D}_{R}=1$ and will then cross the axis at $\mathcal{D}_{R}\left(p_{1}\right)$. Since $f_{0}\left(p_{1}\right)$ is the maximum of $f_{0}$ and $p_{1} \neq 0$, we have $\mathcal{D}_{R}\left(p_{1}\right)<0$. As $\mu_{R}$ is increased it will cross the real axis at $\mathcal{D}_{R}\left(p_{0}\right)$. If $\mathcal{D}_{R}\left(p_{0}\right)>0$ then $\mathcal{C}_{\mathcal{D}}$ will have wrapped around the origin once. As $\mu_{R}$ increases $\mathcal{C}_{\mathcal{D}}$ will cross the line $\mathcal{D}_{I}=0$ (the real axis) at $\mathcal{D}_{R}\left(p_{2}\right)$ and then finish at $\left(\mathcal{D}_{R}, \mathcal{D}_{I}\right)=(1,0)$ as $\mu_{R} \rightarrow \infty$. Therefore, if

$$
\mathcal{D}_{R}\left(p_{2}\right)<0 \quad \text { and } \quad \mathcal{D}_{R}\left(p_{0}\right)>0
$$

$\mathcal{C}_{\mathcal{D}}$ will wrap around the origin twice and the winding number, $M$, is 2 . If, on the other hand, (83) is not satisfied, then $\mathcal{C}_{\mathcal{D}}$ can only wrap around the origin once and the winding number is 1 . As the winding number changes from 1 to 2 , a zero of $\mathcal{D}$ moves from the lower-half plane to the upper-half plane indicating a zero of $\mathcal{D}(\mu)$ with $\operatorname{Im}(\mu)>0$. Therefore, when (83) is satisfied, the spatially homogeneous solution is unstable. A similar argument follows when $p_{1}>p_{2} ;(83)$ is seen to be a condition for instability whenever $p_{1} \neq 0, \mathcal{D}_{R}\left(p_{2}\right) \neq 0$, and $D_{R}\left(p_{0}\right) \neq 0$.

Next, we take $p_{1} \neq 0, p_{1}<p_{2}, \mathcal{D}_{R}\left(p_{0}\right)=0\left(p_{0}=0\right)$, and $\mathcal{D}_{R}\left(p_{2}\right) \neq 0$. As before, $\mathcal{C}_{\mathcal{D}}$ starts on the real axis at $\mathcal{D}_{R}=1$ and crosses the axis at $\mathcal{D}_{R}\left(p_{1}\right)<0$. It next crosses the line $\mathcal{D}_{I}=0$ at the origin, since $\mathcal{D}_{R}\left(p_{0}\right)=0$, and then again at $\mathcal{D}_{R}\left(p_{2}\right) \neq 0$. It follows from (74) that $\mathcal{D}_{I}^{\prime \prime}\left(p_{0}\right)=0$; this means that $\theta_{c}=0$ if $\mathcal{D}^{\prime \prime}\left(p_{0}\right)>0$ and $\theta_{c}=\pi$ if $\mathcal{D}^{\prime \prime}\left(p_{0}\right)<0$. Thus, we see that if

$$
\mathcal{D}_{R}\left(p_{2}\right)<0 \quad \text { and } \quad \mathcal{D}^{\prime \prime}\left(p_{0}\right)>0
$$

$\mathcal{C}_{\mathcal{D}}$ wraps around the origin twice and $M=2$. If (84) is not satisfied then $M=1$, and therefore (84) is the condition for instability. A similar argument holds for $p_{1}>p_{2}$; hence, the condition for instability is given by (84) whenever $p_{1} \neq 0, p_{0}=0$ and $\mathcal{D}_{R}\left(p_{2}\right) \neq 0$. An argument comparable to the one above shows the condition for instability is given by

$$
\mathcal{D}_{R}\left(p_{0}\right)>0 \quad \text { and } \quad \mathcal{D}^{\prime \prime}\left(p_{2}\right)<0
$$

whenever $p_{1} \neq 0, p_{2}=0$ and $\mathcal{D}_{R}\left(p_{0}\right) \neq 0$.

If we compare the expressions for $\mathcal{D}_{R}\left(p_{i}\right)$ and $\mathcal{D}_{R}^{\prime \prime}(0)((82)$ and (76)), we find the condition for instability in the case of (83), (84), and (85) is given by (80) and (81). Finally, we mention that it can be shown, using similar methods to those above, that (80) and (81) is also the condition for instability when $p_{1}=0$. Figure 2 shows an example of a $f_{0}(p)$ which has two peaks. The corresponding $\mathcal{C}_{\mathcal{D}}$ is displayed in figure 3 and clearly shows that $\mathcal{C}_{\mathcal{D}}$ wraps around the origin twice indicating that $f_{0}(p)$ is unstable. The physical meaning of the instability will be explained in section 6 .

\section{Water bags}

We can gain some appreciation of the nonlinear dynamics of the kinetic equation by applying the water-bag model of DePackh [18]. We let the distribution function be piecewise constant in some domain in phase space and find governing equations for the boundaries.

We look for a weak solution of (55) of the form

$$
f(x, p, t)=\sum_{k=1}^{M} a_{k}\left[H\left(p-p_{k}^{-}(x, t)\right)-H\left(p-p_{k}^{+}(x, t)\right)\right]
$$

where $H(p)$ is the Heaviside function, namely

$$
H(p)= \begin{cases}1 & p>0 \\ 0 & p \leqslant 0\end{cases}
$$




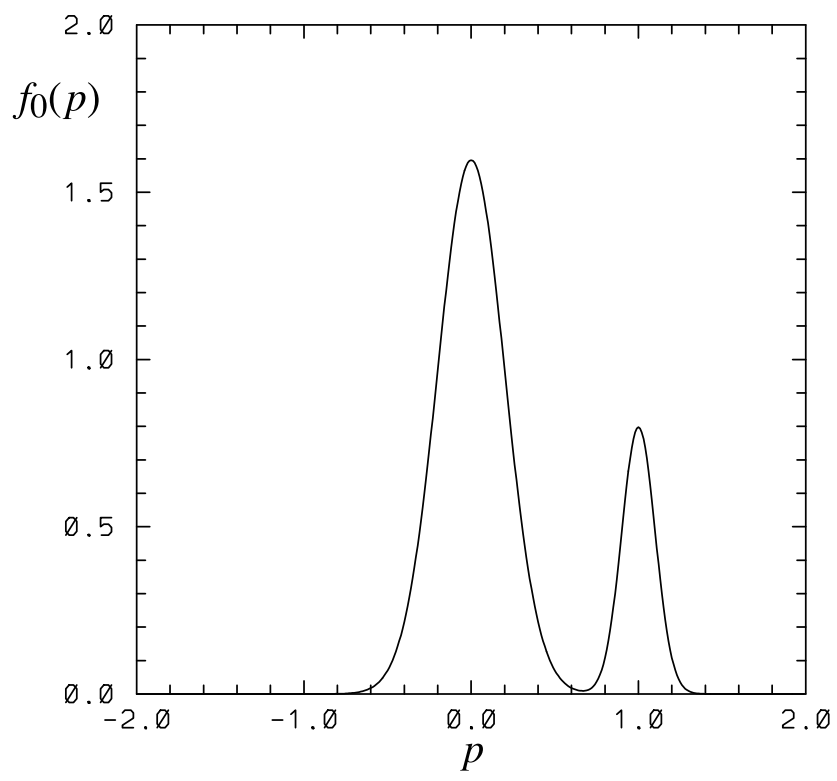

Figure 2. A distribution function with two maxima that is unstable.

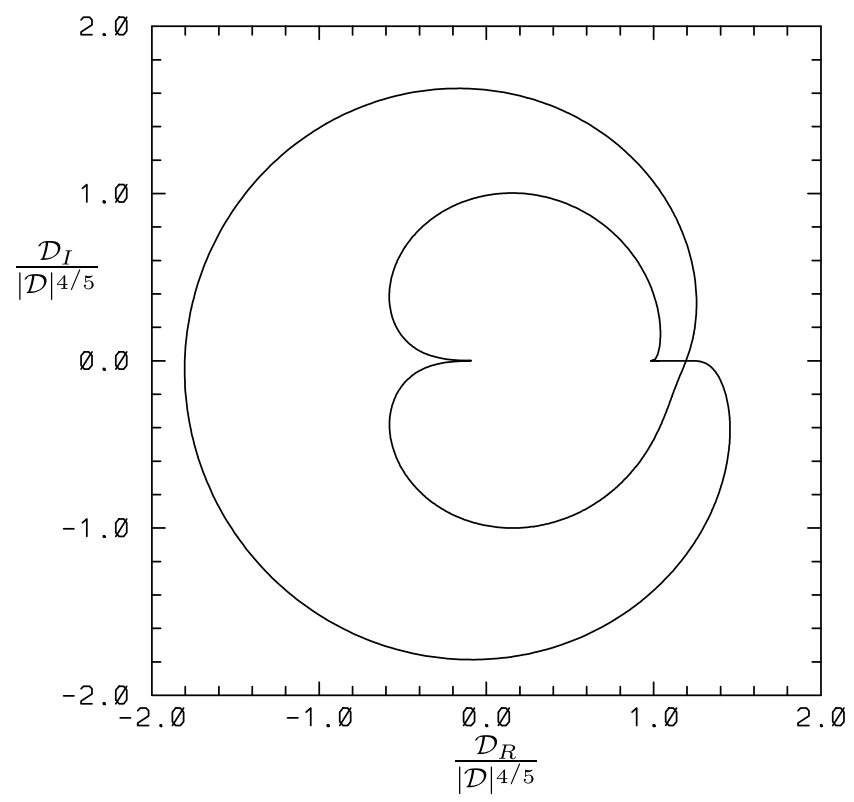

Figure 3. The curve $\mathcal{C}_{\mathcal{D}}$ for the distribution function shown on figure 2. It wraps around the origin twice which indicates an instability.

and $a_{k} \geqslant 0$. We consider $p_{k}^{ \pm}(x, t)$ to be single-valued functions of $x$ and without loss of generality we take $p_{k}^{+}>p_{k}^{-}$. In this case, we have

$$
n(x, t)=\sum_{k=1}^{M} a_{k}\left[p_{k}^{+}(x, t)-p_{k}^{-}(x, t)\right] .
$$


The choice of the $a_{k}$ and the $p_{k}^{ \pm}$is constrained since $n(x, 0)=1$. We also have

$$
j(x, t)=\frac{1}{2} \sum_{k=1}^{M} a_{k}\left[\left(p_{k}^{+}\right)^{2}(x, t)-\left(p_{k}^{-}\right)^{2}(x, t)\right] .
$$

If we substitute (86) into (55), we find

$$
\begin{aligned}
\sum_{k=1}^{M} a_{k} \delta\left(p-p_{k}^{+}\right) & {\left[\frac{\partial p_{k}^{+}}{\partial t}+\kappa(p-j) \frac{\partial p_{k}^{+}}{\partial x}-\kappa p \frac{\partial j}{\partial x}\right] } \\
& -a_{k} \delta\left(p-p_{k}^{-}\right)\left[\frac{\partial p_{k}^{-}}{\partial t}+\kappa(p-j) \frac{\partial p_{k}^{-}}{\partial x}-\kappa p \frac{\partial j}{\partial x}\right]=0 .
\end{aligned}
$$

As pointed out by DePackh, this equation must vanish term-by-term; thus we find the system of $2 M$ equations in conservation law form:

$$
\frac{\partial p_{k}^{ \pm}}{\partial t}+\kappa \frac{\partial}{\partial x}\left[\frac{1}{2}\left(p_{k}^{ \pm}\right)^{2}-p_{k}^{ \pm} j\right]=0 .
$$

It follows from (89) that if $n(x, 0)=1$ then $n(x, t)=1$ for $t>0$. It is also convenient to compute the turbulent kinetic energy; in the present situation, (52) simplifies to

$$
\theta(x, t)=\frac{\kappa^{2}}{2} \int(p-\bar{p})^{2} f \mathrm{~d} p .
$$

The simplest case is $M=1$. It follows, in view of the constraint $n(x, t)=1$, that

$$
p_{1}^{+}(x, t)-p_{1}^{-}(x, t)=\Delta
$$

where $\Delta$ is a constant independent of $x$ and $t$. Therefore, we have

$$
j(x, t)=\frac{1}{2}\left(p_{1}^{+}(x, t)+p_{1}^{-}(x, t)\right)
$$

and

$$
\frac{\partial j}{\partial t}-\kappa j \frac{\partial j}{\partial x}=0
$$

which we recognize as Burgers' equation. This indicates that $p_{1}^{ \pm}$can form shocks in finite time.

The next case we consider is $M=2$. We let $\boldsymbol{P}=\left(p_{1}^{-}, p_{1}^{+}, p_{2}^{-}, p_{2}^{+}\right)^{T}$ and then write (89) as

$$
\frac{\partial \boldsymbol{P}}{\partial t}+\kappa \mathcal{A} \frac{\partial \boldsymbol{P}}{\partial x}=0
$$

where

$$
\mathcal{A}=\left(\begin{array}{cccc}
p_{1}^{-}-j-a_{1}\left(p_{1}^{-}\right)^{2} & -a_{1} p_{1}^{-} p_{1}^{+} & a_{2} p_{1}^{-} p_{2}^{-} & -a_{2} p_{1}^{-} p_{2}^{+} \\
a_{1} p_{1}^{-} p_{1}^{+} & p_{1}^{+}-j-a_{1}\left(p_{1}^{+}\right)^{2} & a_{2} p_{1}^{+} p_{2}^{-} & -a_{2} p_{1}^{+} p_{2}^{+} \\
a_{1} p_{1}^{-} p_{2}^{-} & -a_{1} p_{1}^{+} p_{2}^{-} & p_{2}^{-}-j+a_{2}\left(p_{2}^{-}\right)^{2} & -a_{2} p_{2}^{-} p_{2}^{+} \\
a_{1} p_{1}^{-} p_{2}^{+} & -a_{1} p_{1}^{+} p_{2}^{+} & a_{2} p_{2}^{-} p_{2}^{+} & p_{2}^{+}-j-a_{2}\left(p_{2}^{+}\right)^{2}
\end{array}\right) .
$$

The hyperbolicity of this system is determined by the eigenvalues of $\mathcal{A}$. If the eigenvalues are real, the system is hyperbolic; if the eigenvalues are complex the system is elliptic and the initial value problem is ill-posed. In the water-bag model, for a fixed $x$, the distribution function is supported over the intervals $I_{1}=\left[p_{1}^{-}, p_{1}^{+}\right]$and $I_{2}=\left[p_{2}^{-}, p_{2}^{+}\right]$. Below we shall prove that if the intervals overlap, the eigenvalues of $\mathcal{A}$ are real, and that if there is a sufficiently large distance between the centers of $I_{1}$ and $I_{2}$, the eigenvalues of $\mathcal{A}$ are complex. 
To prove this claim it is useful to use the following variables:

$p_{2}^{-}=p_{1}^{-}+c-e \quad p_{2}^{+}=p_{1}^{+}+c+e \quad$ and $\quad a_{1}=(1-y) / \Delta$

where $0 \leqslant y \leqslant 1$ and $\Delta=p_{1}^{+}-p_{1}^{-}$. The value of $a_{2}$ is found from (87). The distance between the centres of $I_{1}$ and $I_{2}$ is $|c|$. The intervals will overlap when $|c|<|\mathrm{e}+\Delta|$. A calculation shows that (93) is hyperbolic when

$$
c^{2}<C_{c r}^{2}
$$

where

$$
C_{c r}^{2}=\frac{(\Delta+2 e)^{2}-4 e y(\Delta+\mathrm{e})}{4 y(1-y)} .
$$

Therefore we see that if $|c|$ is sufficiently large (93) is elliptic. On the other hand, it is possible to show that

$$
|\mathrm{e}+\Delta|<C_{c r}
$$

implying that if the intervals overlap (93) is hyperbolic. When the intervals overlap the distribution function has a 'single maximum'. Furthermore, the distribution function must have 'two maxima' in the ill-posed case. These results are consistent with the results of section 5 .

Consider (93), with periodic boundary conditions on the interval $[-\pi, \pi]$, and the following initial data:

$$
\begin{aligned}
& p_{1}^{ \pm}(x, 0)= \pm 0.2 \\
& p_{2}^{-}(x, 0)=0.9+\varepsilon \sin (x) \\
& p_{2}^{+}(x, 0)=1.0+\varepsilon \sin (x)
\end{aligned}
$$

where we take $a_{1}=2$ and $a_{2}=2$. We see that for this choice of initial data, with $\varepsilon=0, f(x, p, 0)$ is a spatially uniform distribution solution of (93) with 'two maxima'. Furthermore, (95) reveals this solution to be unstable. The time evolution of a small perturbation of this solution is studied by numerically solving (93), the initial data is given by (96) with $\varepsilon=0.05$.

Equation (93), with initial data given by (96), is ill-posed and cannot be solved numerically without some care. We chose to solve (89) by centre differencing in space. To advance in time, a fourth order Runge-Kutta-Merson scheme was used. We filter after each time step using the filter developed by Krasny [19].

The initial conditions are plotted in figure 4. Figure 5 shows the distribution function at $t=4.25$ which is close to the last time we can solve (93). It appears a singularity is forming.

To analyse the singularity, we examine the Fourier coefficients of $p_{i}^{ \pm}(x, t)$. It is well known that if

$$
f(x, t)) \approx\left[x-\left(x_{0}+\mathrm{i} \rho(t)\right)\right]^{\alpha(t)-1}
$$

the Fourier coefficients decay as

$$
\hat{f}(k) \approx c k^{-\alpha(t)} \mathrm{e}^{-\rho(t) k} .
$$




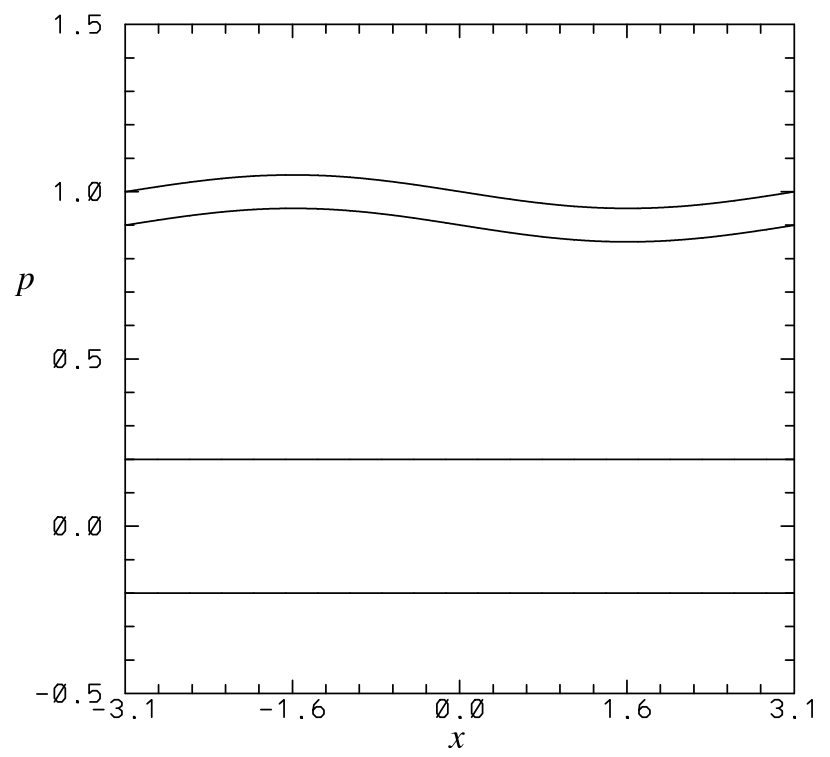

Figure 4. The initial values used for the water-bag problem. $f_{0}(x, p, 0)$ is given by (96) with $a_{1}=2$ and $a_{2}=2$. The lines are, from bottom to top, respectively, $p_{1}^{-}, p_{1}^{+}, p_{2}^{-}$, and $p_{2}^{+}$. If a portion of the phase space $(x, p)$ is not between the lines $p_{1}^{-}(x, 0)$ and $p_{1}^{+}(x, 0)$, or between the lines $p_{2}^{-}(x, 0)$ and $p_{2}^{+}(x, 0)$, then $f(x, p, 0)$ is zero.

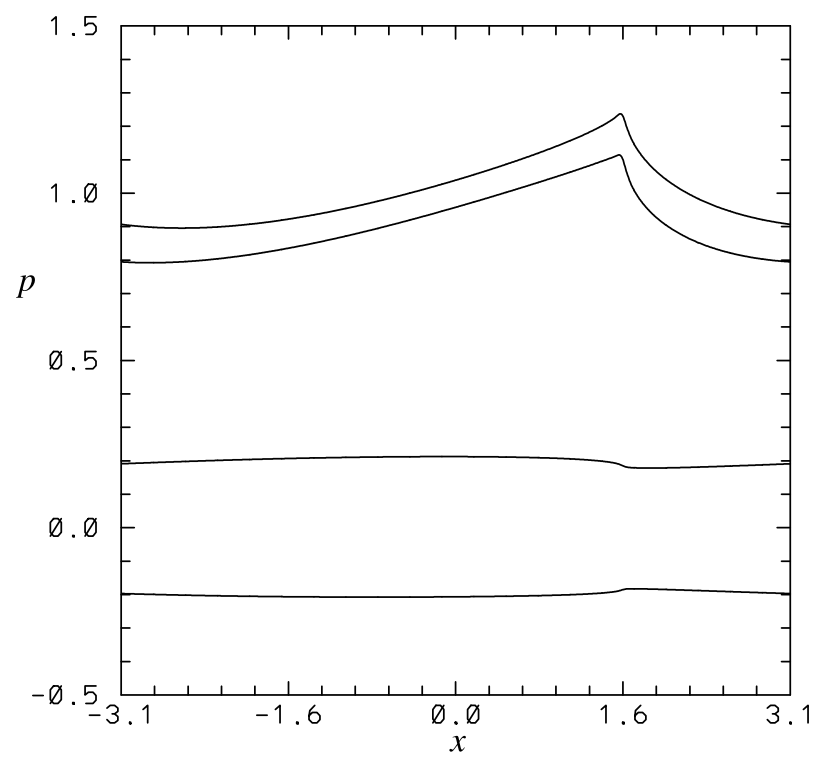

Figure 5. The water-bag problem at $t=4.25$ for the initial conditions shown in figure 4 .

There are several methods to estimate $\alpha$ and $\rho$ in the literature; see, for example, [19-21]. Here we use a variant of these methods which is explained below. From (97) it follows that

$$
\frac{\hat{f}(k)}{\hat{f}(\ell)}=\left(\frac{k}{\ell}\right)^{-\alpha} \mathrm{e}^{-\rho(k-\ell)}
$$


which can be written as

$$
y_{k}=-\alpha-\rho z_{k}
$$

where

$$
y_{k}=\frac{\log (\hat{f}(k) / \hat{f}(\ell))}{\log (k / \ell)} \quad \text { and } \quad z_{k}=\frac{k-\ell}{\log (k / \ell)} .
$$

To estimate $\alpha$ and $\rho$, we plot $y_{k}$ against $z_{k}$ for a fixed $\ell$. The slope gives $\rho$ and the $y$ intercept provides $\alpha$. We pick $\ell=3$ and measure the slope for various $k$. We find that our estimates of $\alpha$ and $\rho$ depend slightly on $k$; for $5 \leqslant k \leqslant 15, \alpha$ and $\rho$ vary approximately 5\%. The average values of $\alpha$ and $\rho$ for $k \in[5,15]$ will be used as our estimate.

It is found, for the above computation, that $\alpha$ and $\rho$ are approximately the same (within 5\%) for each component of $\boldsymbol{P}$. $\rho$ vanishes at $t=t_{c} \approx 4.27$ and over the time interval $\left[3.5, t_{c}\right] \rho$ decreases linearly. On the other hand, $\alpha$ is approximately constant having the value

$$
\alpha=\frac{3}{2} \pm 0.04 \text {. }
$$

A plot of $\log _{10}\left|\hat{p}_{2}^{+}(k)\right|$ verses $\log _{10} k$ is shown in figure 7 along with the line whose slope is -1.5 . This supports the estimate that $\alpha=1.5$. This value of $\alpha$ indicates a square root singularity. At $t=t_{c}$, the profiles of $p_{i}^{ \pm}$have the following form:

$$
p_{1}^{ \pm} \sim \operatorname{sgn}(x) \sqrt{|x|} \quad \text { and } \quad p_{2}^{ \pm} \sim \sqrt{|x|} .
$$

Figure 6 shows the turbulent kinetic energy intensifying at the point the solution becomes singular.

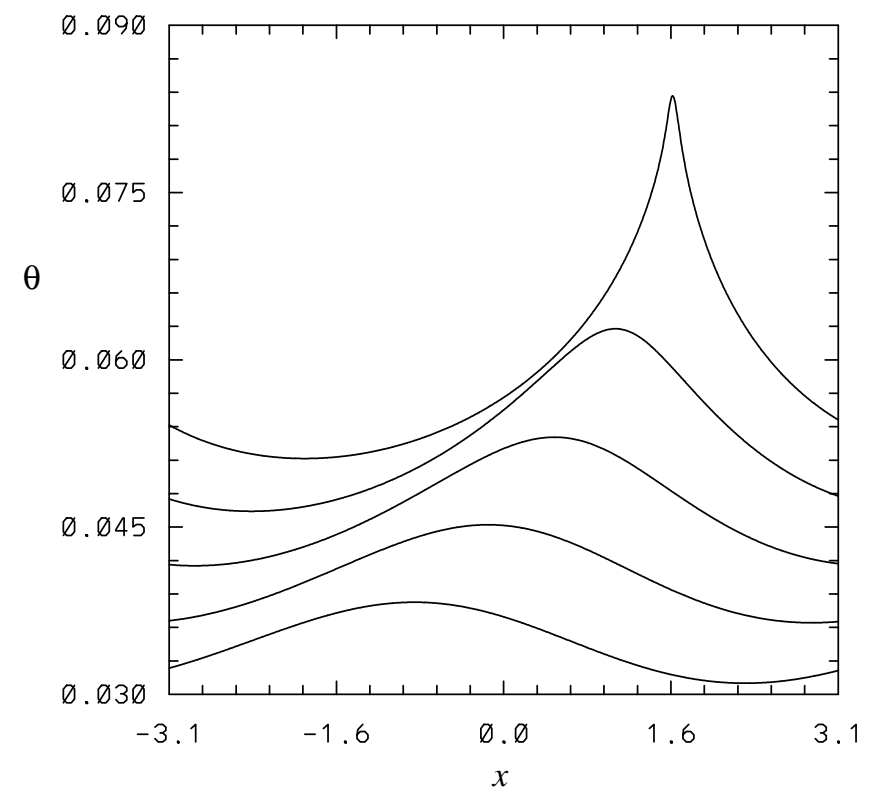

Figure 6. The turbulent kinetic energy, $\theta$, as a function of $x$ for various times using the waterbag model. The times shown are $t=0.85,1.70,2.55,3.40$, and 4.25. Time increases upward and subsequent snapshots are shifted upwards by 0.003 for clarity. 


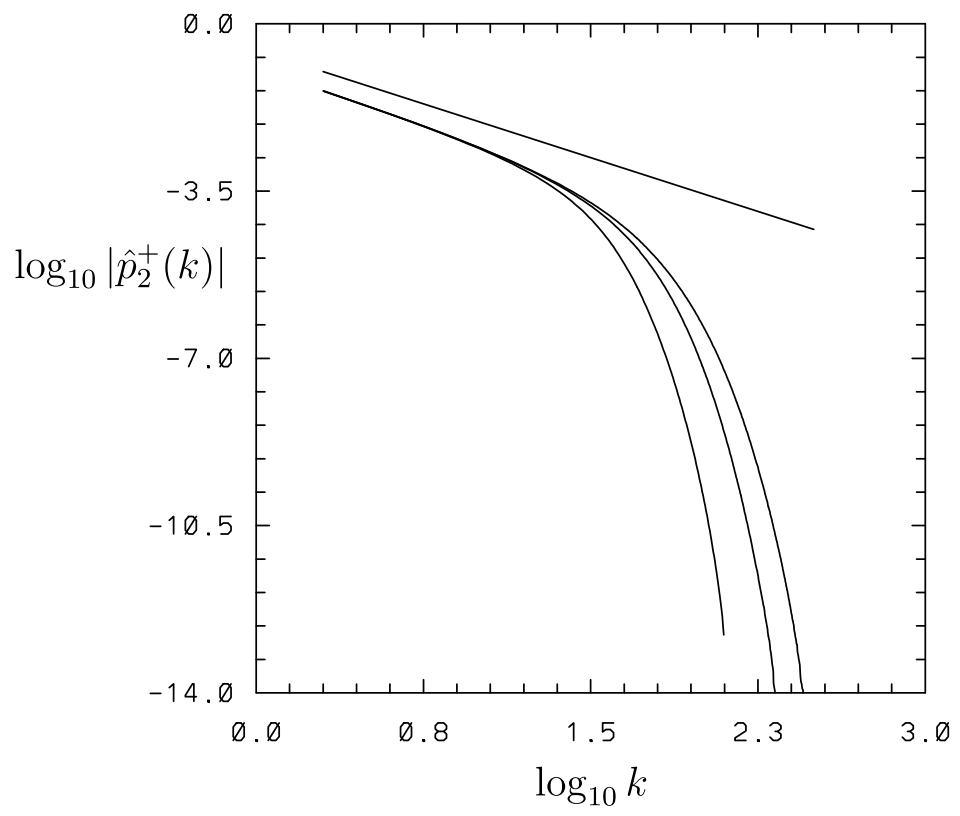

Figure 7. $\log -\log$ plot of the Fourier coefficients of $p_{2}^{+}$at $t=4.25$ for different values of $N$, the number of points used in the discretization. The curves from left to right have $N=256$, 512 , and 1024. The slope of the straight line is -1.5 .

Next, we consider the following initial data:

$$
\begin{aligned}
& p_{1}^{ \pm}(x, 0)= \pm 0.2 \\
& p_{2}^{ \pm}(x, 0)= \pm 0.1+\varepsilon \sin (x)
\end{aligned}
$$

with $a_{1}=2, a_{2}=1$, and $\varepsilon=0.05$. Equation (93) is well-posed with this initial data; generically we expect this solution to form shocks in finite time in a similar fashion to Burgers' equation. We present numerical solutions for (93) with the initial data given by (98) in figures 8 and 9. Figure 8 shows the initial condition and figure 9 shows the solution at $t=37$, just before it forms a shock.

Remark. There has been recent work on weak solutions of the Vlasov-Poisson equation which have some similarities to this work. Majda [22] has numerically studied weak solutions of the Vlasov-Poisson equation of the form

$$
f(z(\alpha, t))=\left|\frac{\mathrm{d} z}{\mathrm{~d} t}\right|^{-1} \delta_{z(\alpha, t)} \quad \text { where } z(\alpha, t)=(x(\alpha, t), v(\alpha, t)) .
$$

This is called an electron sheet solution. There is an analytical solution of this form due to Dziurzynski [23] which has a finite time singularity. After the singular time, the numerical work of Majda shows that the analytical solution is no longer valid; the electron sheet ceases to be the graph of the single-valued function and the electric field loses smoothness past the singular time. This work has been extended and generalized by Majda et al [24]. They present explicit examples with charge concentration and non-unique weak solutions. They also show that the various regularizations of the Vlasov-Poisson give different weak solutions. Zheng and Majda [25] have provided a natural definition for a weak solution of the Vlasov-Poisson equation. They have proved that the Vlasov-Poisson equation has globally weak solutions with measures as initial data. 


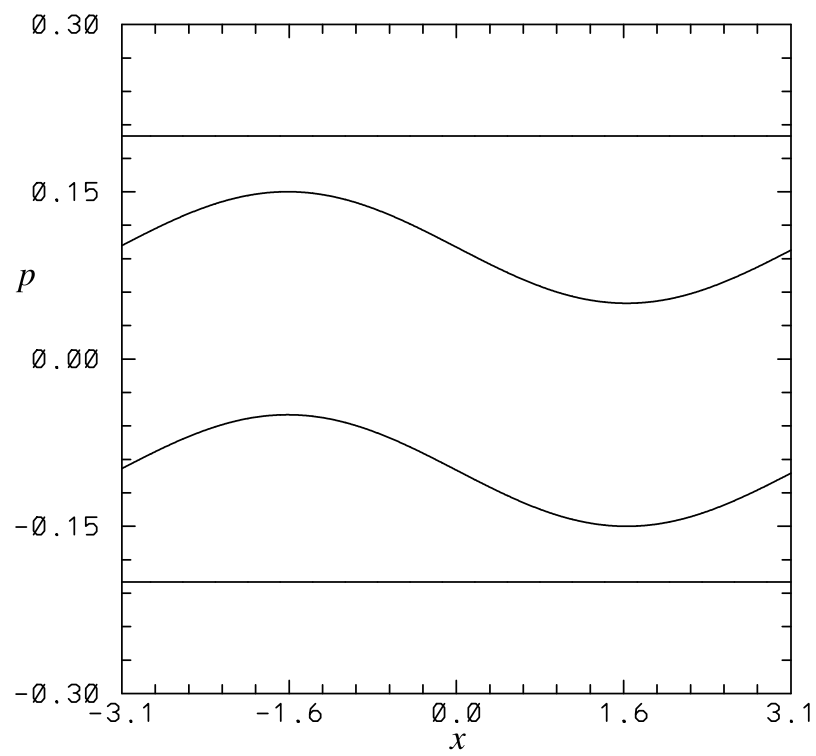

Figure 8. The initial value conditions for the water-bag problem given by equation (98) with $a_{1}=2$ and $a_{2}=1$. Starting at the bottom the lines are $p_{1}^{-}, p_{2}^{-}, p_{2}^{+}$, and $p_{1}^{+}$.

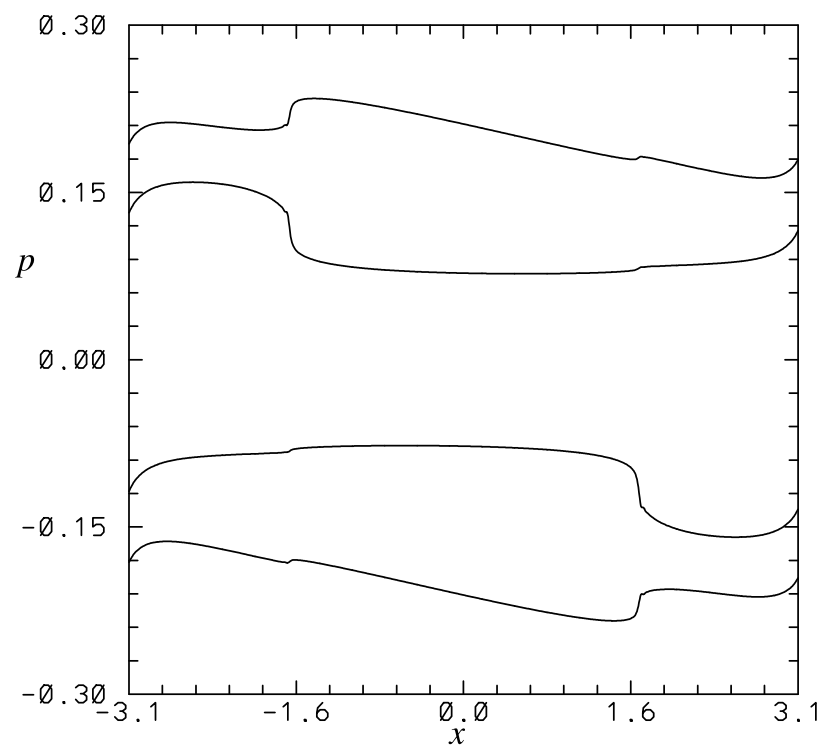

Figure 9. Same as figure 8 except $t=37$.

The water-bag solutions are also weak solutions of a kinetic equation. The numerical evidence shows that they also form singularities in finite time. It may be possible for one to use the ideas developed in $[22,24,25]$ to construct weak solutions past the singular time. It would also be interesting to examine what effect the type of regularization has on the nature of weak solutions to our kinetic equation. 


\section{Discussion}

We have developed a kinetic equation which, we have argued, describes the probability distribution, $f$, of the impulse density in a turbulent fluid. It was established that any spatially homogeneous distribution function $(f=f(p))$ is a solution of the kinetic equation. We interpret this solution to represent a fluid in a state of homogeneous turbulence.

Batchelor [26] (see p 185) points out that experimental observations suggest that the high wave-number components of turbulence are unevenly distributed in space. This would indicate that the spatially uniform turbulent state in some sense is unstable. We have found several situations where the spatially uniform solution evolves into a spatially nonuniform solution.

In one case it was found that when the distribution function had two maxima and one minimum it was possible for the spatially uniform solution to be linearly unstable. Numerical solutions of the water-bag model indicate that cusp-like singularities will form in finite time. On the other hand, even when the spatially homogeneous solution is linearly stable we observe shock-like singularity. Therefore, under rather a wide variety of initial conditions, the spatially homogeneous solution will evolve so that the turbulence is no longer spatially uniform.

For example, consider an initially quiescent fluid with a fine grid placed into it. We shall take the grid to be parallel to the $y z$-plane and moving in the positive $x$-direction, which we will denote as the stream-wise direction. On the far-right-hand side of the grid, the fluid is at rest; as the grid moves through the liquid it will disturb the liquid behind it. We expect the region of fluid immediately behind the grid to be somewhat homogeneously turbulent [26].

It will require a force in the $x$-direction to move the grid; therefore, we expect the grid to cause the appearance of a high impulse region in the stream-wise direction. It is possible that the distribution of impulse in the coarsening volume will be similar to that shown in figure 2 which was seen to be unstable. Consequently, the apparent spottiness of the spatial distribution of the turbulent energy could be the result of this instability.

To understand the small scales of physical turbulence one must include viscosity. Therefore, it would be interesting to include viscosity in the kinetic formulation. As of yet it is not clear exactly how one would go about this.

\section{Summary}

In this paper we have shown that the impulse equation has a gauge freedom which allows it to be generalized. We have demonstrated that the divergence-free projection operator can be written in terms of a continuous distribution of vortex dipoles which have a finite self-induced velocity. This splits the projection operator into a local part and a nonlocal part.

It is then shown that for a particular choice of gauge, the force density can be written in terms of the nonlocal part of the divergence-free projection. Based on this observation, a Vlasov description of the impulse form of the Euler equation is proposed. This kinetic equation is seen to have the Euler equation as a solution for special initial data. It is proposed that for other initial data, the Vlasov equation provides a coarse-grained description of an inviscid turbulent flow.

It is also shown that the Vlasov equation has nontrivial solutions in one space dimension. Furthermore, we demonstrate that spatially homogeneous distribution functions with single maxima are linearly stable, and that those with two maxima can be unstable. The time evolution of the unstable solutions are studied and we observe that singularities form. In our case these singularities are associated with localization of the turbulent kinetic energy. 


\section{Acknowledgments}

I express my gratitude to Brenda Brown for reading over this manuscript. I thank Chris Anderson, Robert Krasny, Robert Pego and Michael Weinstein for fruitful comments and Yann Brenier for suggesting the water-bag model. I give special thanks to Russel Caflisch for numerous very helpful discussions and for his interest and support of this work. I also give thanks to one of the referees for his useful comments. A large portion of this work was performed in the Department of Mathematics at UCLA where it was supported, in part, by ARPA under URI grant number \# N00014092-J-1890.

\section{References}

[1] Sagdeev R Z, Moiseev S S, Tur A V and Yanovskii V V 1986 Problems of the theory of strong turbulence and topological solitons Nonlinear Phenomena in Plasma Physics and Hydrodynamics ed R Z Sagdeev (Moscow: Mir)

[2] Kuz'min G A 1983 Ideal incompressible hydrodynamics in terms of the vortex momentum density Phys. Lett. 96A 88

[3] Oseledets V I 1989 On a new way of writing the Navier-Stokes equation: The Hamiltonian formalism Russ. Math. Surveys $\mathbf{4 4} 210$

[4] Tur A V and Yanonsky V V 1993 Invariants in dissipationless hydrodynamic media J. Fluid. Mech. 24867

[5] Buttke T F 1993 Velicity methods: Lagrangian numerical methods which preserve the Hamiltonian structure of incompressible fluid flow Vortex Flows and Related Numerical Methods ed J T Beale, G H Cottet and S Huberson (Dordrecht: Kluwer)

[6] Buttke T F and Chorin A F 1993 Turbulence calculations in magnetization variables Appl. Num. Math. 12 47

[7] Maddocks J H and Pego R L 1995 An unconstrained Hamiltonian formulation for incompressible fluid flow Commun. Math. Phys. 170207

[8] Krasny R 1989 A vortex-dipole sheet model for a wake 1989 Phys. Fluids A 1173

[9] Chorin A J 1994 Vortex phase transitions in $2 \frac{1}{2}$ dimensions J. Stat. Phys. 76835

[10] Batchelor G K 1967 An Introduction to Fluid Dynamics (Cambridge: Cambridge University Press)

[11] Brenier Y 1989 A Vlasov-Poisson type formulation of the Euler equations INRIA Report 1070

[12] Roberts P H 1972 A Hamiltonian theory for weakly interacting vortices Mathematica 19169

[13] Chefranov S G 1987 Dynamics of point-vortex dipoles and spontaneous singularities in three dimensional turbulent flows Sov. Phys.-JETP 6685

[14] Caflisch R E and Smereka P 1994 Unpublished notes

[15] Liboff R L 1969 Introduction to the Theory of Kinetic Equations (New York: Wiley)

[16] Landau L 1946 On the vibrations of the electronic plasma J. Phys. (USSR) 1025

[17] Nicholson D R 1982 Introduction to Plasma Theory (New York: Wiley)

[18] DePackh D C 1962 The water-bag model of a sheet electron beam J. Electr. Contr. 13417

[19] Krasny R 1986 A study of singularity formation in a vortex sheet by the point-vortex approximation J. Fluid. Mech. 16765

[20] Sulem C, Sulem P L and Frisch U 1983 Tracing complex singularities with spectral methods J. Comput. Phys. 50138

[21] Caflisch R E 1993 Singularity formation for complex solutions of the 3D incompressible Euler equations Physica 67D 1

[22] Majda G 1993 On singular solutions of the Vlasov-Poisson equation Vortex Flows and Related Numerical Methods ed J T Beale, G H Cottet and S Huberson (Dordrecht: Kluwer)

[23] Dziurzynski R S 1987 Patches of electrons and electron sheets for the 1D Vlasov-Poisson equation $P h D$ Dissertation Department of Mathematics, University of California, Berkeley

[24] Majda A J, Majda G and Zheng Y 1993 Concentrations in the one-dimensional Vlasov-Poisson equations I: temporal development and non-unique weak solutions in the single component case Physica D $\mathbf{7 4} 268$

[25] Zheng Y and Majda A J 1994 Existence of global weak solutions of one-component Vlasov-Poisson and Fokker-Plank-Poisson systems in one space dimension with measures as initial data Commun. Pure Appl. Math. 471365

[26] Batchelor G K 1953 The Theory of Homogeneous Turbulence (Cambridge: Cambridge University Press) 